\title{
Alkaloids from Gelsemium elegans
}

You-Kai Xu, ${ }^{\ddagger}$ Sheng-Ping Yang, ${ }^{\dagger}$ Shang-Gao Liao, ${ }^{\dagger}$ Hua Zhang, ${ }^{\dagger}$ Li-Ping Lin, ${ }^{\dagger}$ Jian Ding, ${ }^{\dagger}$ and Jian-Min Yue ${ }^{* \dagger}$

State Key Laboratory of Drug Research, Shanghai Institute of Materia Medica, Shanghai Institutes for Biological Sciences, Chinese Academy of Sciences, 555 Zu Chong Zhi Road, Zhangjiang Hi-Tech Park, Shanghai, 201203, People's Republic of China and Xishuangbanna Tropical Botanical Garden, Chinese Academy of Sciences, Yunnan, Mengla, 666303, People's Republic of China

\section{Supporting Information}

S1. Selected HMBC and key ROESY correlations of $14 \alpha$-hydroxyelegansamine (2).

S2. Selected HMBC and key ROESY correlations of $14 \alpha$-hydroxygelsamydine (3).

S3. ${ }^{1} \mathrm{H}$ NMR (Varian Mercury-400, $400 \mathrm{MHz}, \mathrm{CDCl}_{3}$ ) data of gelsebanine (1).

S4. ${ }^{13} \mathrm{C}$ NMR (Varian Mercury-400, $100 \mathrm{MHz}, \mathrm{CDCl}_{3}$ ) data of gelsebanine (1).

S5. HSQC (Varian Mercury-400, $400 \mathrm{MHz}, \mathrm{CDCl}_{3}$ ) data of gelsebanine (1).

S6. HMBC (Varian Mercury-400, $400 \mathrm{MHz}, \mathrm{CDCl}_{3}$ ) data of gelsebanine (1).

S7. ROESY (Varian Inova-600, $600 \mathrm{MHz}, \mathrm{CDCl}_{3}$ ) data of gelsebanine (1).

S8. EIMS of gelsebanine (1).

S9. IR spectrum of gelsebanine (1).

S10. UV spectrum of gelsebanine (1).

S11. ${ }^{1} \mathrm{H}$ NMR (Varian Mercury-400, $400 \mathrm{MHz}, \mathrm{CDCl}_{3}$ ) data of $14 \alpha$-hydroxyelegansamine (2).

S12. ${ }^{13} \mathrm{C}$ NMR (Varian Mercury-400, $100 \mathrm{MHz}, \mathrm{CDCl}_{3}$ ) data of $14 \alpha$-hydroxyelegansamine (2).

S13. HSQC (Varian Mercury-400, $400 \mathrm{MHz}, \mathrm{CDCl}_{3}$ ) data of $14 \alpha$-hydroxyelegansamine (2).

S14. HMBC (Varian Mercury-400, $400 \mathrm{MHz}, \mathrm{CDCl}_{3}$ ) data of $14 \alpha$-hydroxyelegansamine (2).

\footnotetext{
* Corresponding author. Tel: +86-21-50806718. Fax: +86-21-50806718. E-mail: jmyue@mail.shcnc.ac.cn.

${ }^{\dagger}$ Shanghai Institute of Materia Medica.

${ }^{\ddagger}$ Xishuangbanna Tropical Botanical Garden.
} 
S15. ROESY (Varian Mercury-400, $400 \mathrm{MHz}, \mathrm{CDCl}_{3}$ ) data of $14 \alpha$-hydroxyelegansamine (2).

S16. EIMS of $14 \alpha$-hydroxyelegansamine (2).

S17. IR spectrum of $14 \alpha$-hydroxyelegansamine (2).

S18 UV spectrum of $14 \alpha$-hydroxyelegansamine (2).

S19. ${ }^{1} \mathrm{H}$ NMR (Varian Mercury-400, $400 \mathrm{MHz}, \mathrm{CDCl}_{3}$ ) data of $14 \alpha$-hydroxygelsamydine (3).

S20. ${ }^{13} \mathrm{C}$ NMR (Varian Mercury-400, $100 \mathrm{MHz}, \mathrm{CDCl}_{3}$ ) data of $14 \alpha$-hydroxygelsamydine (3).

S21. ${ }^{13} \mathrm{CNMR}$ (Varian Mercury-400, $100 \mathrm{MHz}, \mathrm{CDCl}_{3}$ (80\%)+CD $\mathrm{CD}_{3} \mathrm{OD}$ (20\%)) data of $14 \alpha$-hydroxygelsamydine (3).

S22. HSQC (Varian Mercury-400, $400 \mathrm{MHz}, \mathrm{CDCl}_{3}$ ) data of $14 \alpha$-hydroxygelsamydine (3).

S23. HMBC (Varian Mercury-400, $400 \mathrm{MHz}, \mathrm{CDCl}_{3}$ ) data of $14 \alpha$-hydroxygelsamydine (3).

S24. ROESY (Varian Mercury-400, $400 \mathrm{MHz}, \mathrm{CDCl}_{3}$ ) data of $14 \alpha$-hydroxygelsamydine (3).

S25. EIMS of 14 $\alpha$-hydroxygelsamydine (3).

S26. IR spectrum of $14 \alpha$-hydroxygelsamydine (3)

S27. UV spectrum of $14 \alpha$-hydroxygelsamydine (3).

S28. ${ }^{1} \mathrm{H}$ NMR (Varian Mercury-400, $400 \mathrm{MHz}, \mathrm{CDCl}_{3}$ ) data of gelsebamine (4).

S29. ${ }^{13} \mathrm{C}$ NMR (Varian Mercury-400, $100 \mathrm{MHz}, \mathrm{CDCl}_{3}$ ) data of gelsebamine (4).

S30. HSQC (Varian Mercury-400, $400 \mathrm{MHz}, \mathrm{CDCl}_{3}$ ) data of gelsebamine (4).

S31. HMBC (Varian Mercury-400, $400 \mathrm{MHz}, \mathrm{CDCl}_{3}$ ) data of gelsebamine (4).

S32. ROESY (Varian Mercury-400, $400 \mathrm{MHz}, \mathrm{CDCl}_{3}$ ) data of gelsebamine (4).

S33. EIMS of gelsebamine (4).

S34. IR spectrum of gelsebamine (4).

S35. Comparative TLC check of crude alkaloids for gelsebamine (4). 


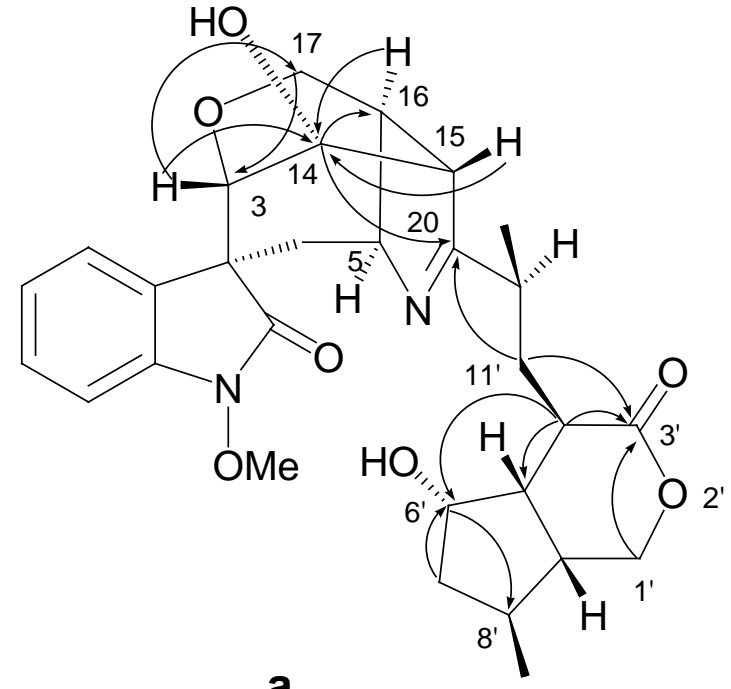

a

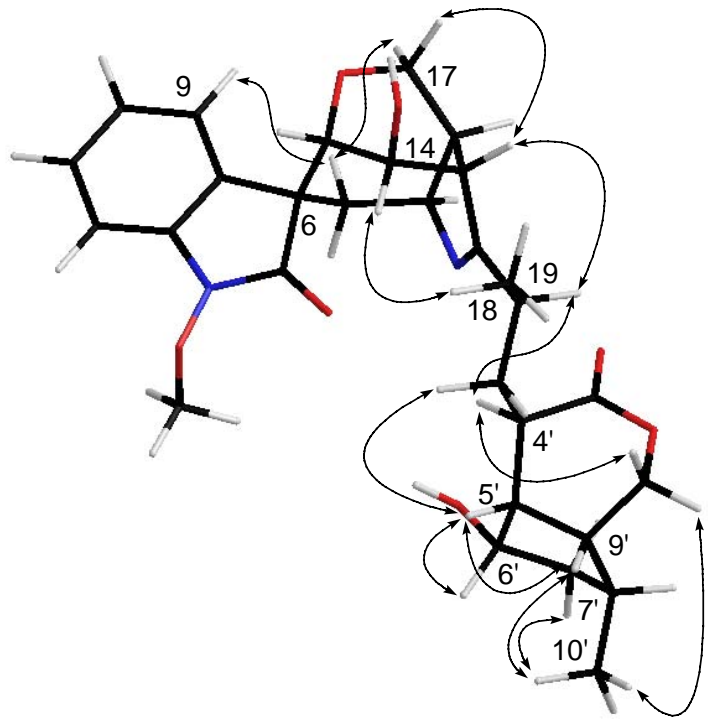

b

Figure S1. Selected HMBC $(\rightarrow)$ and key ROESY $(\leftrightarrow)$ correlations of 14 $\alpha$-hydroxyelegansamine (2). 


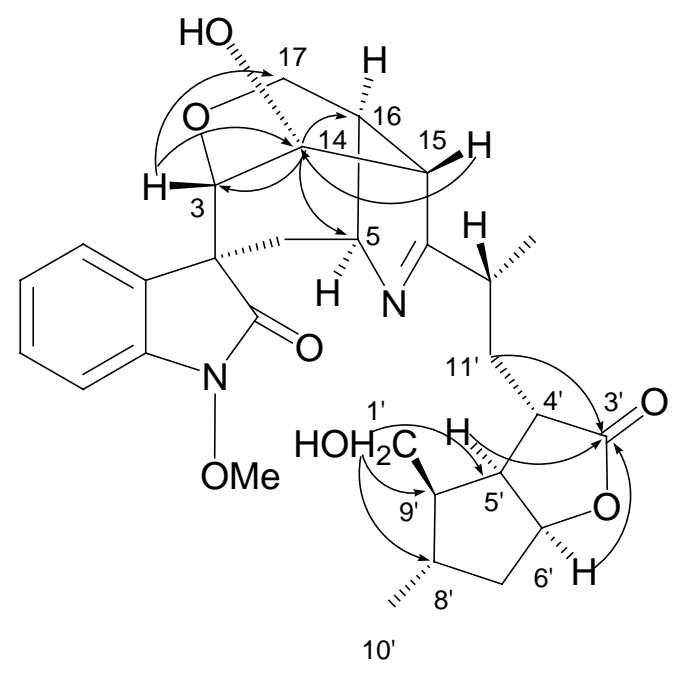

a

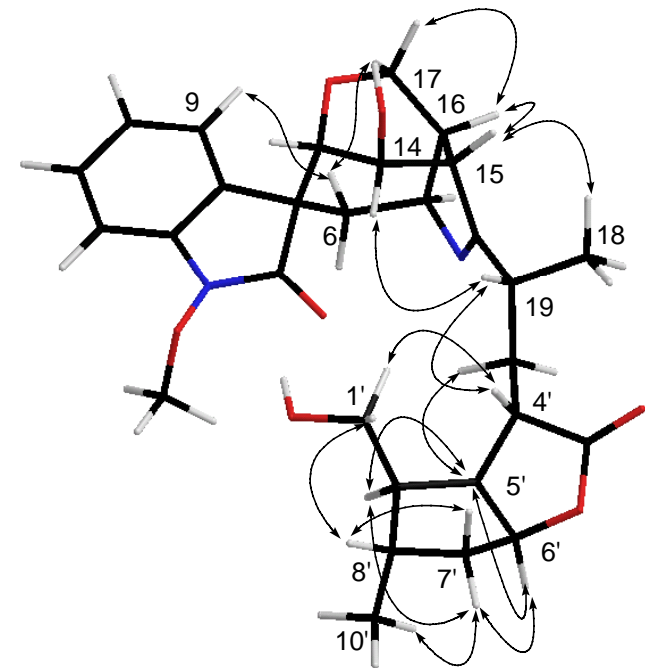

b

Figure S2. Selected HMBC $(\rightarrow)$ and key ROESY $(\leftrightarrow)$ correlations of $14 \alpha$-hydroxygelsamydine (3). 


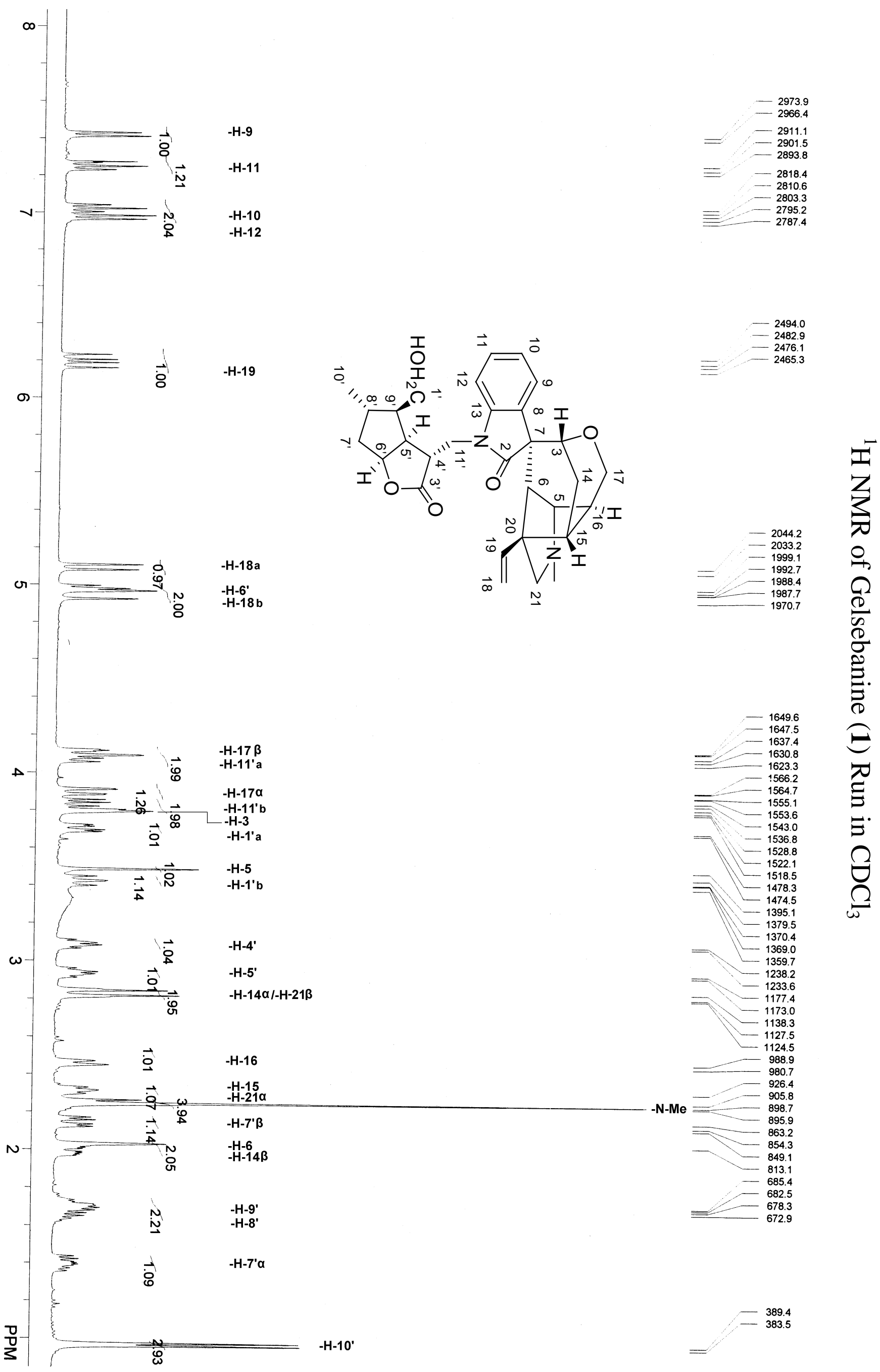




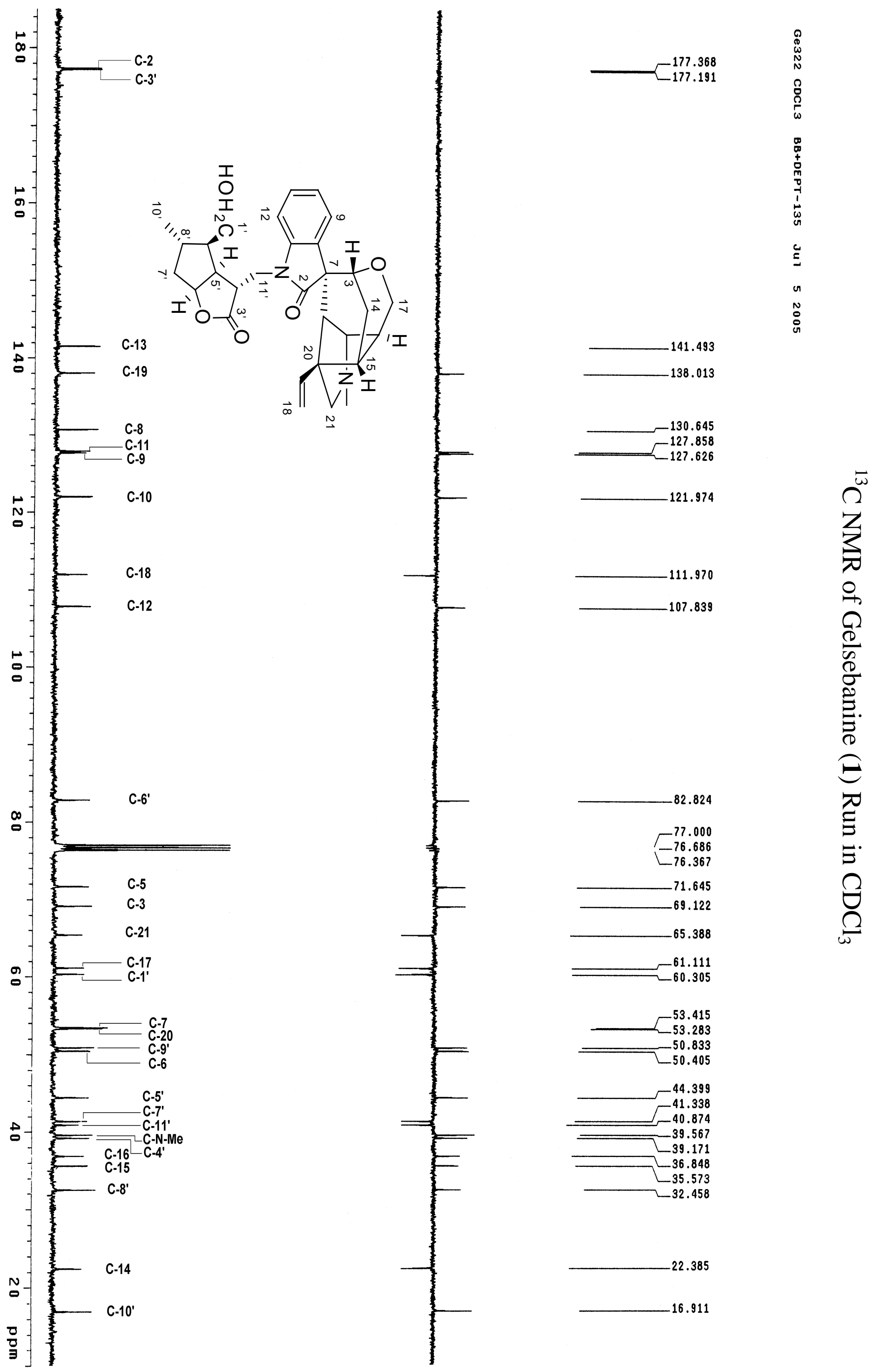




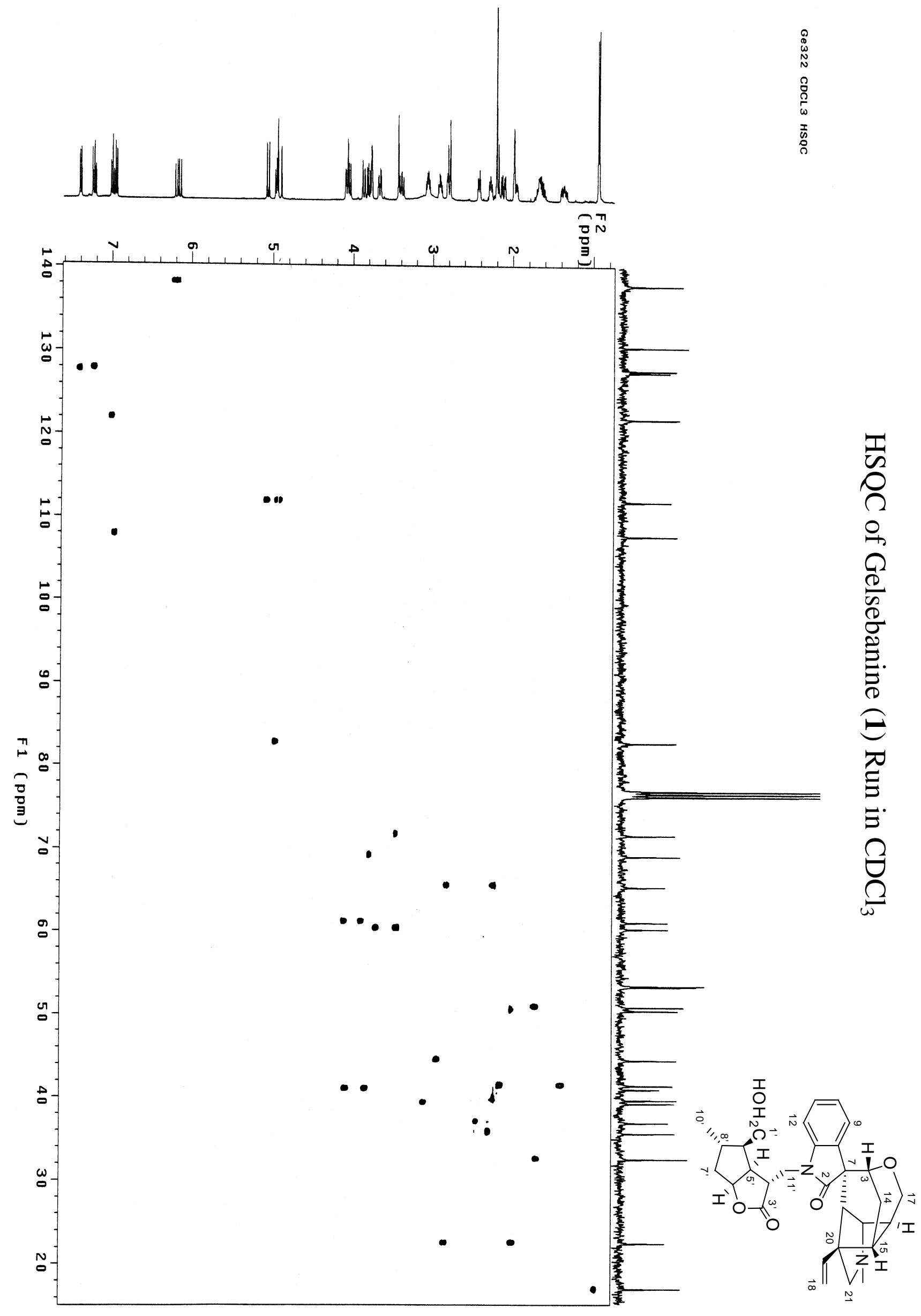




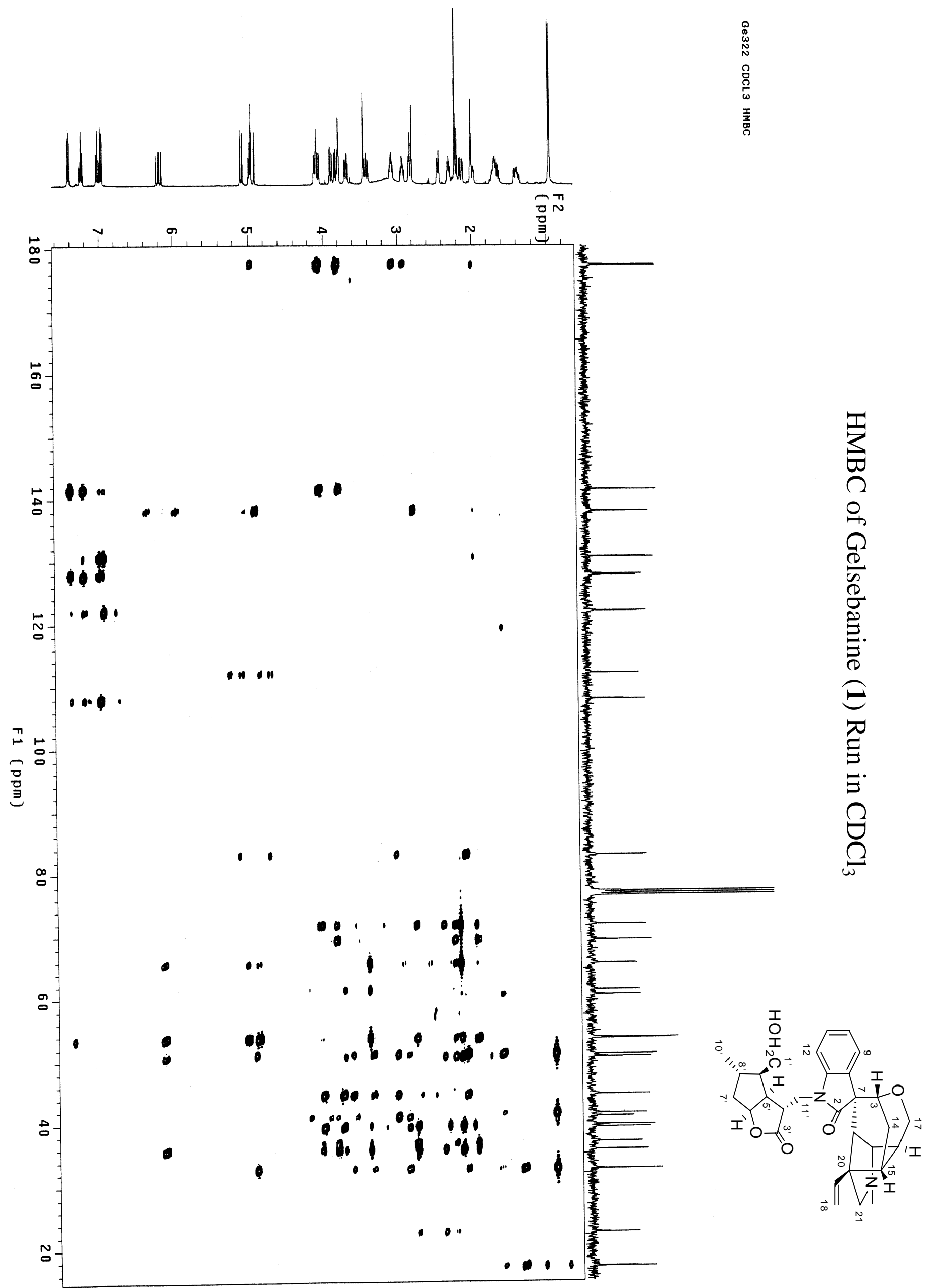




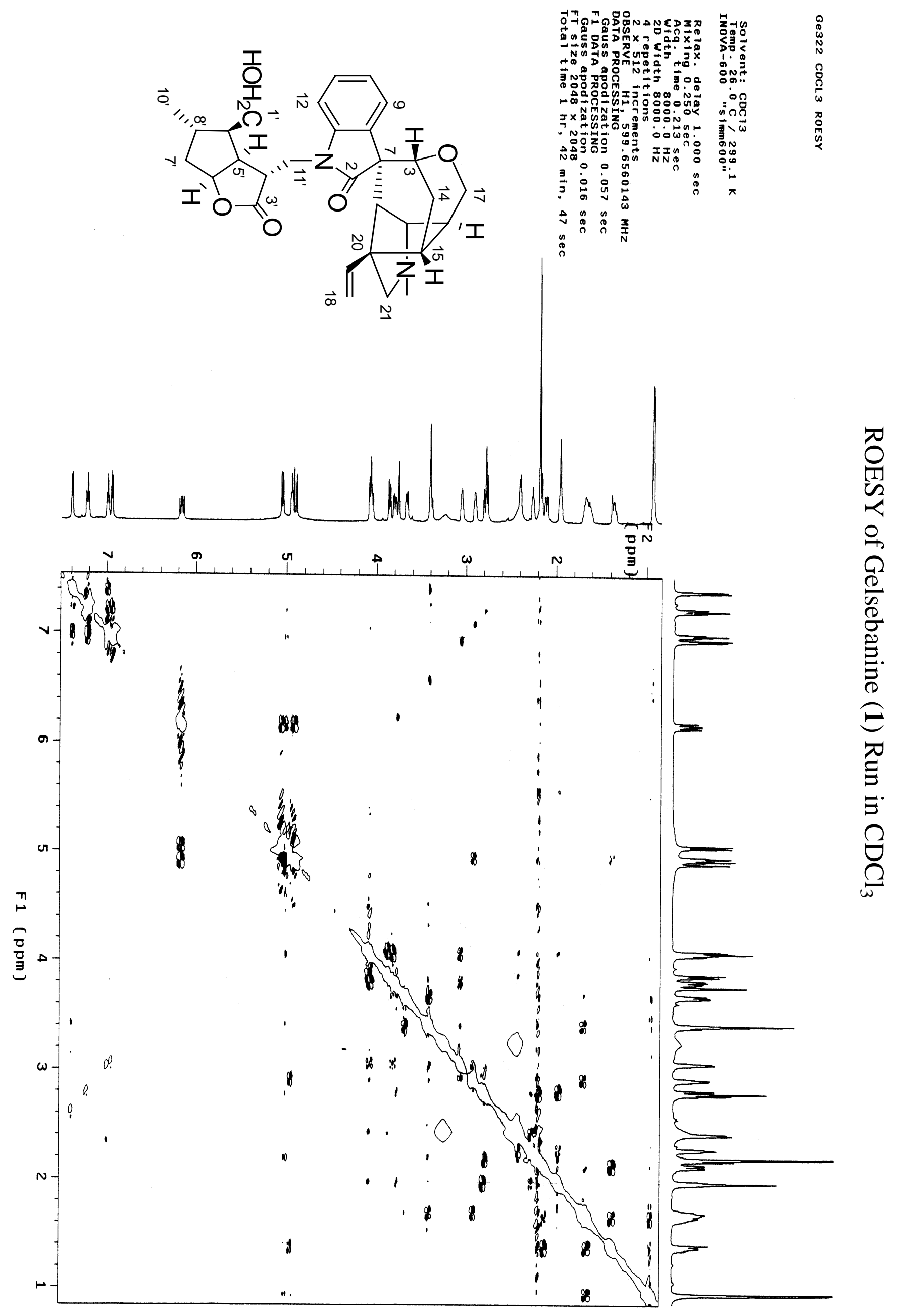




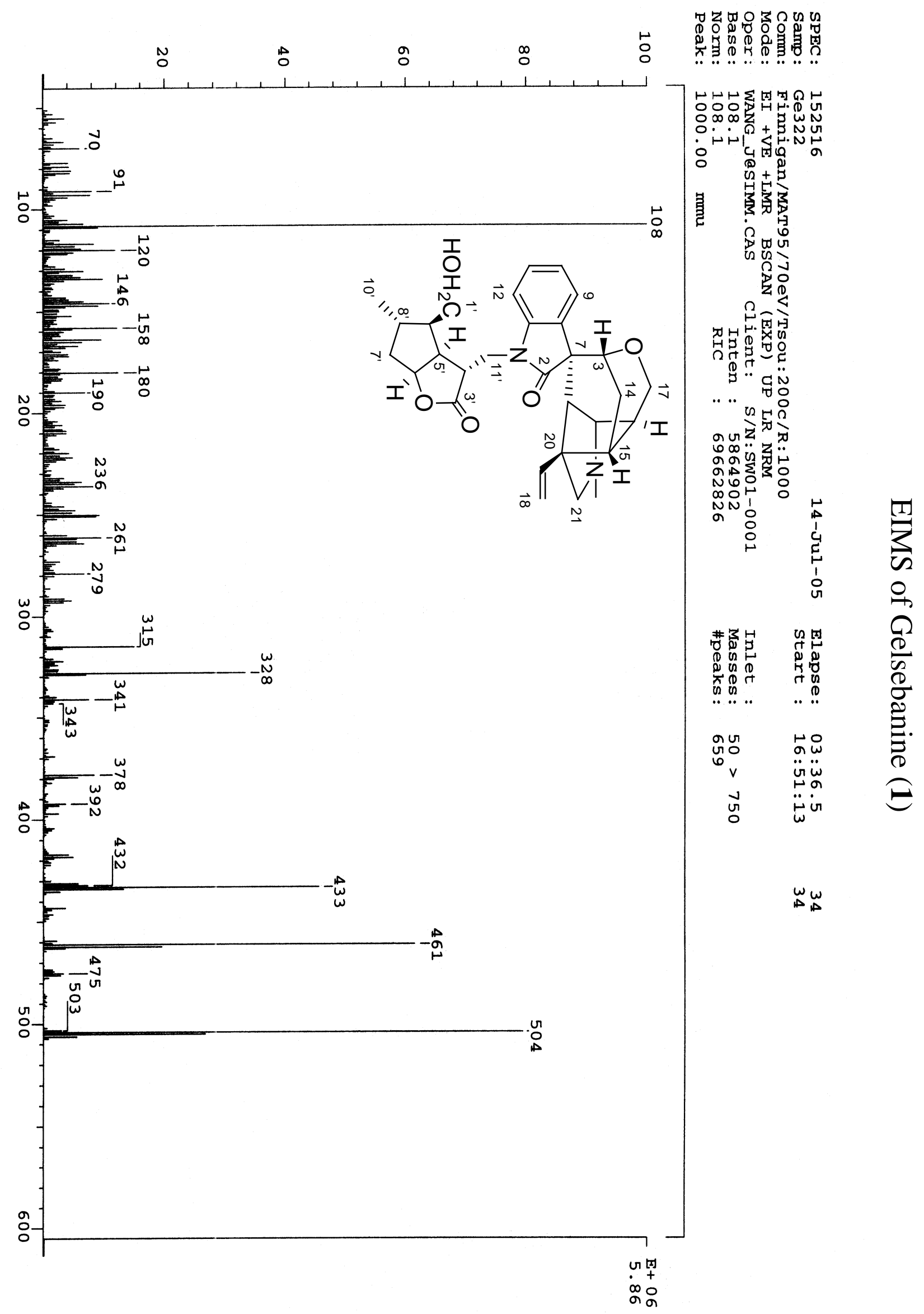




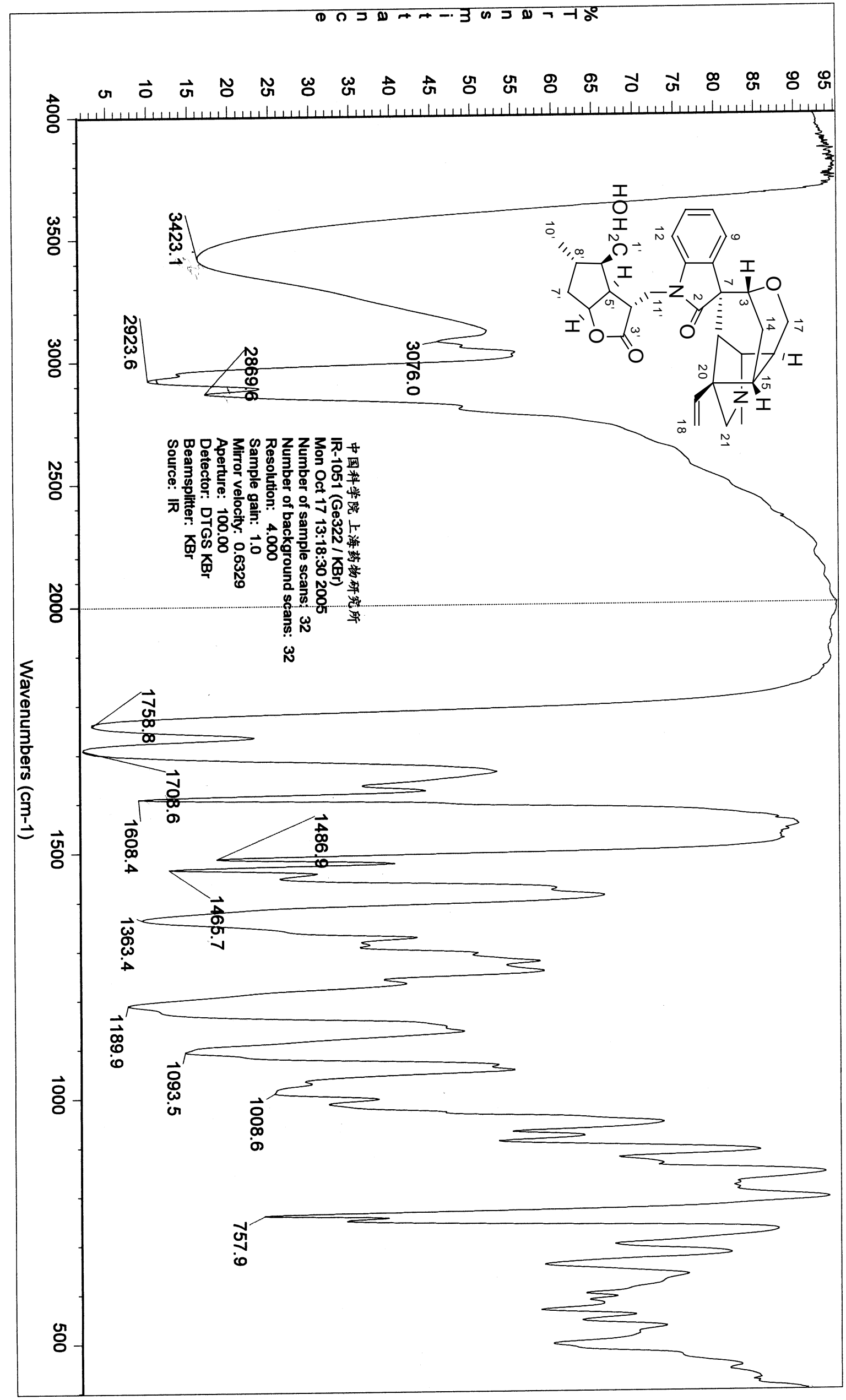

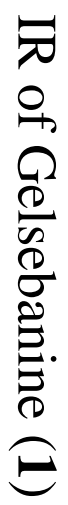



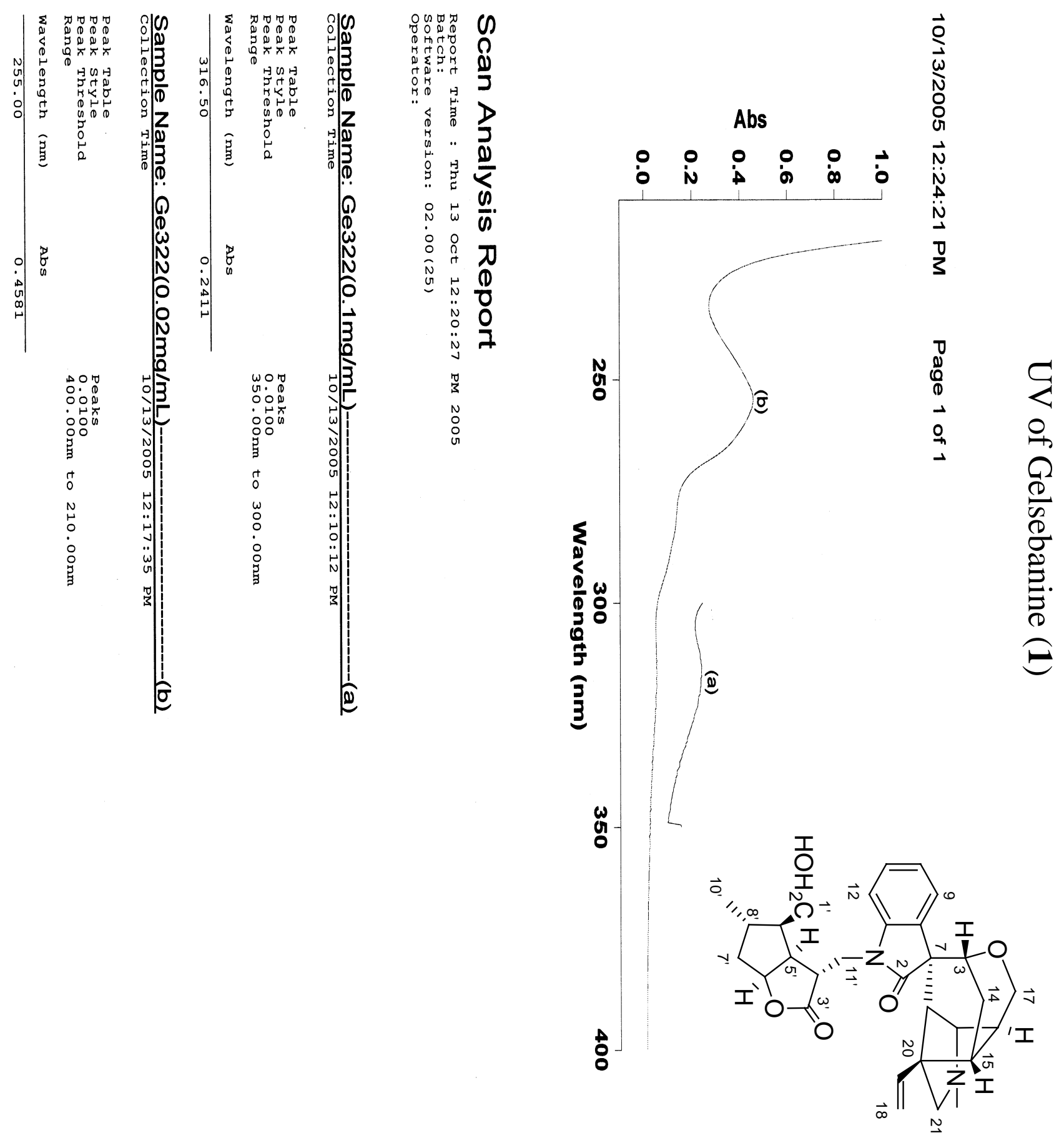


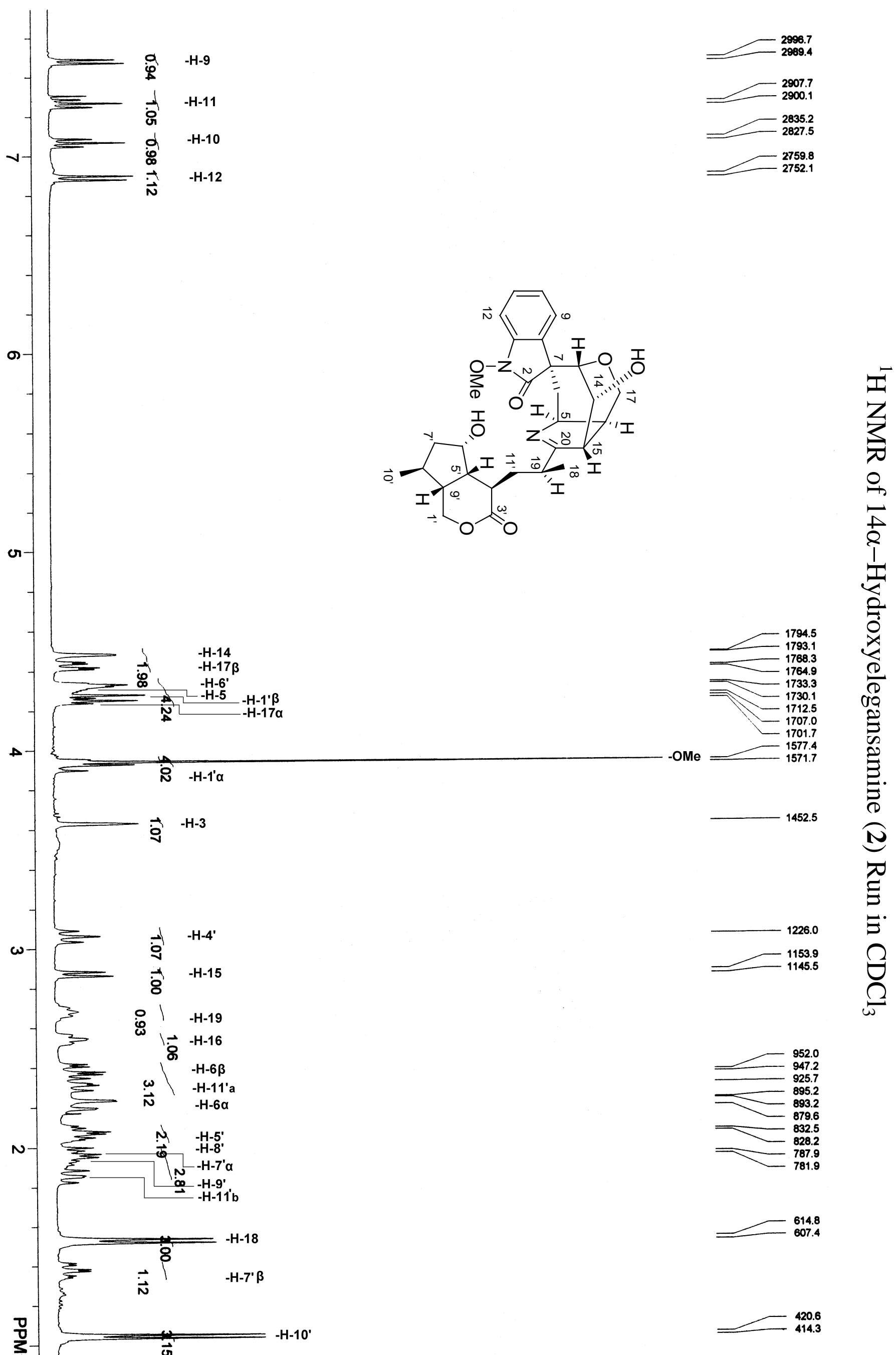



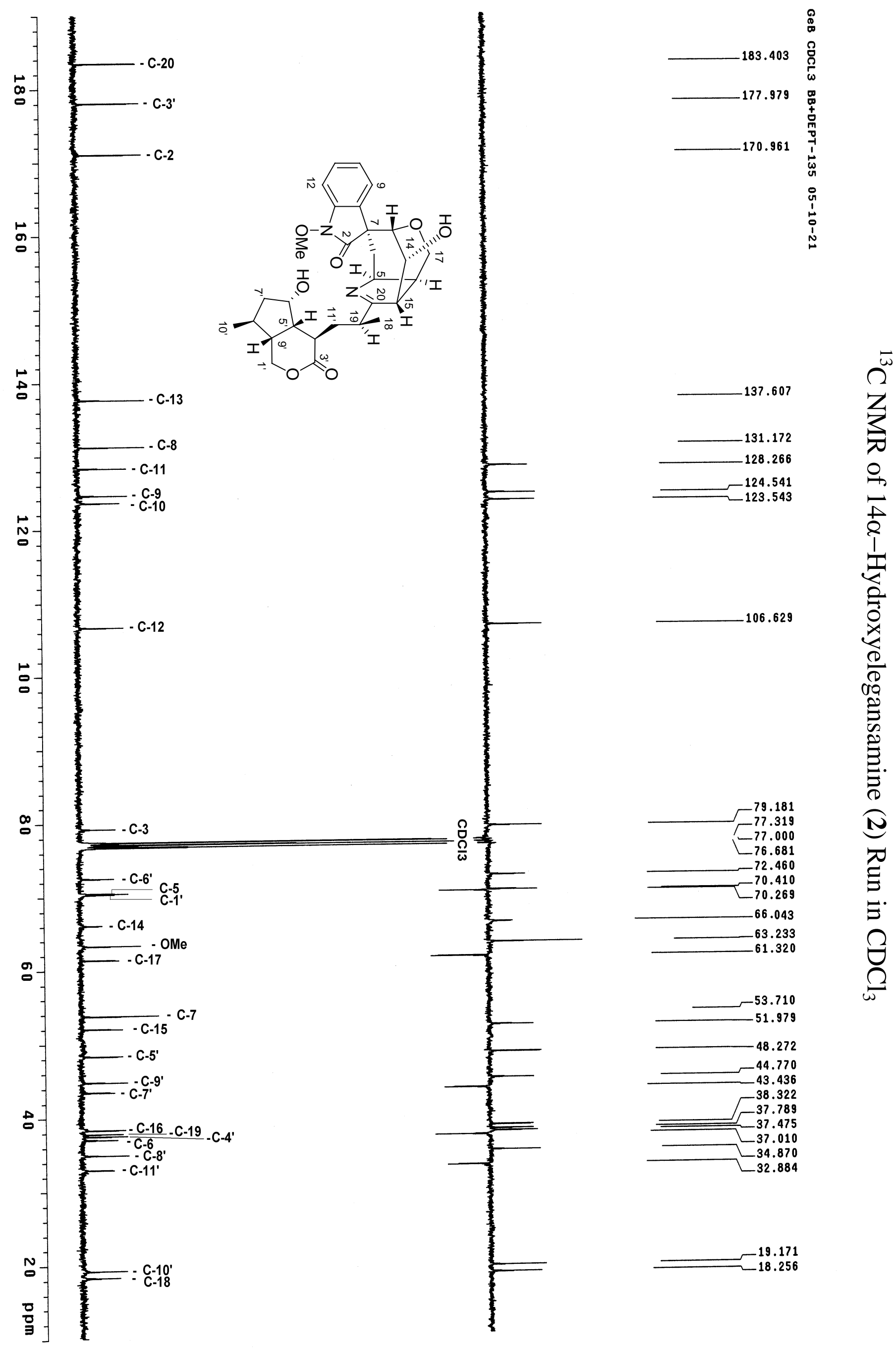


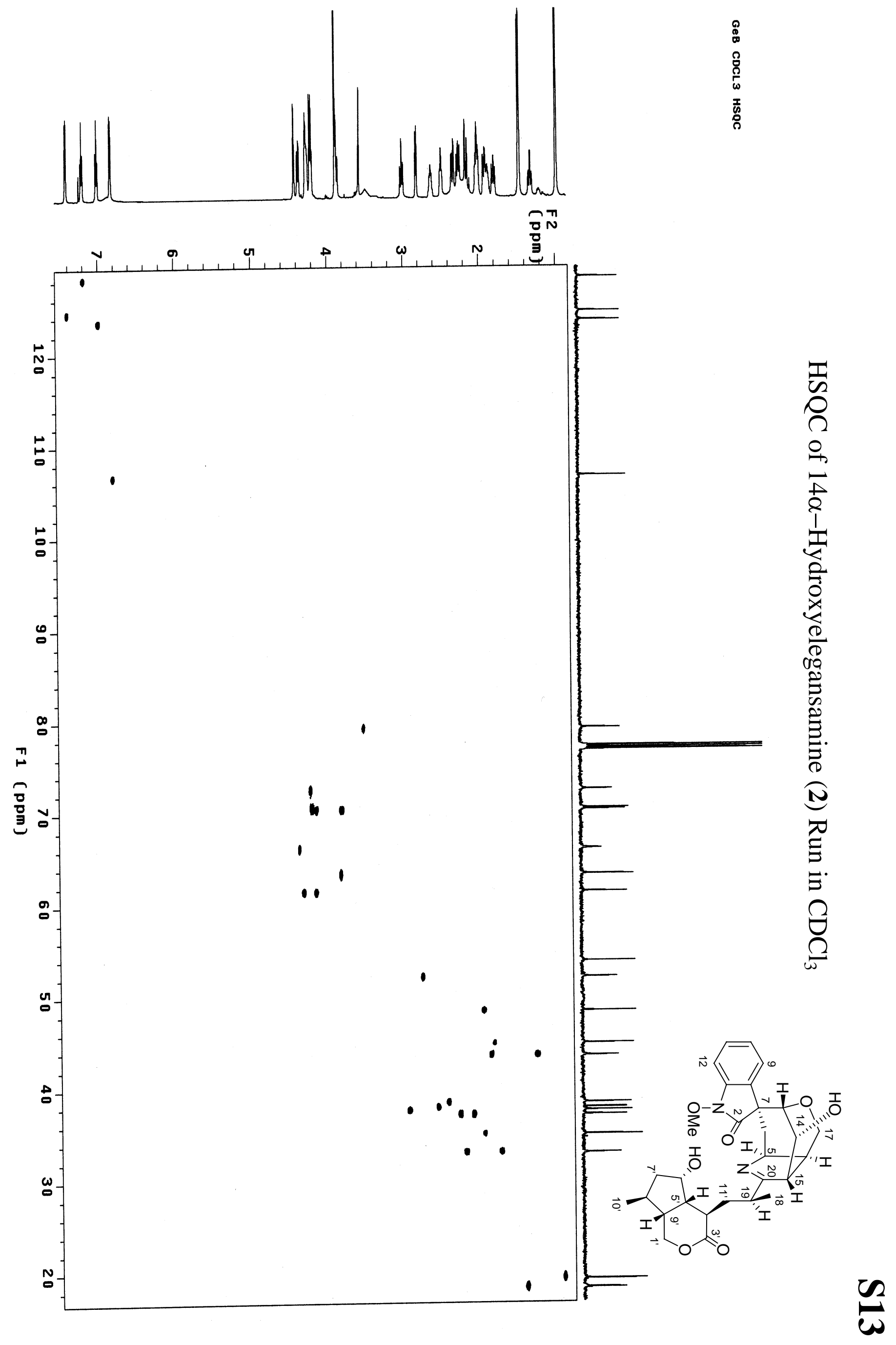




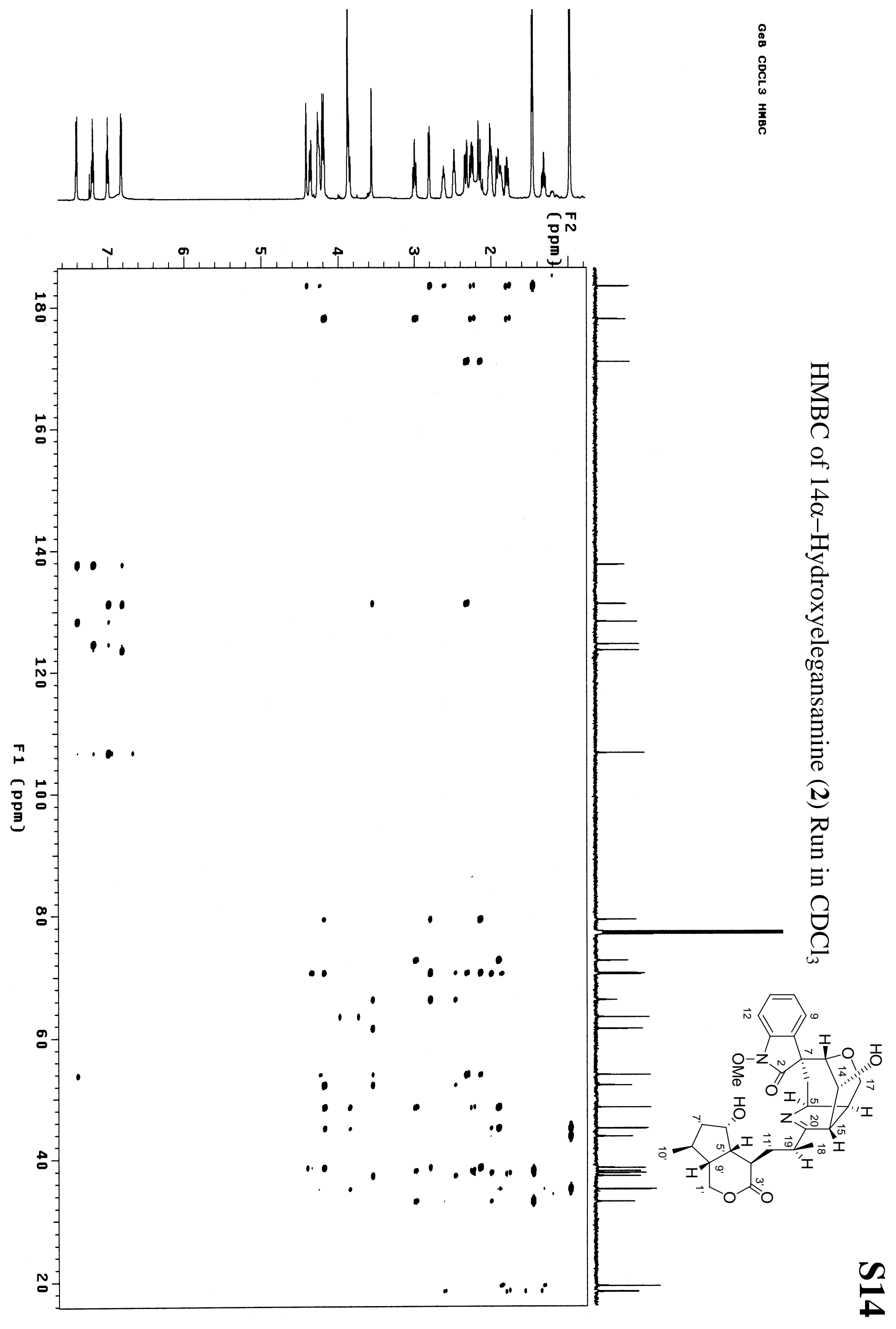




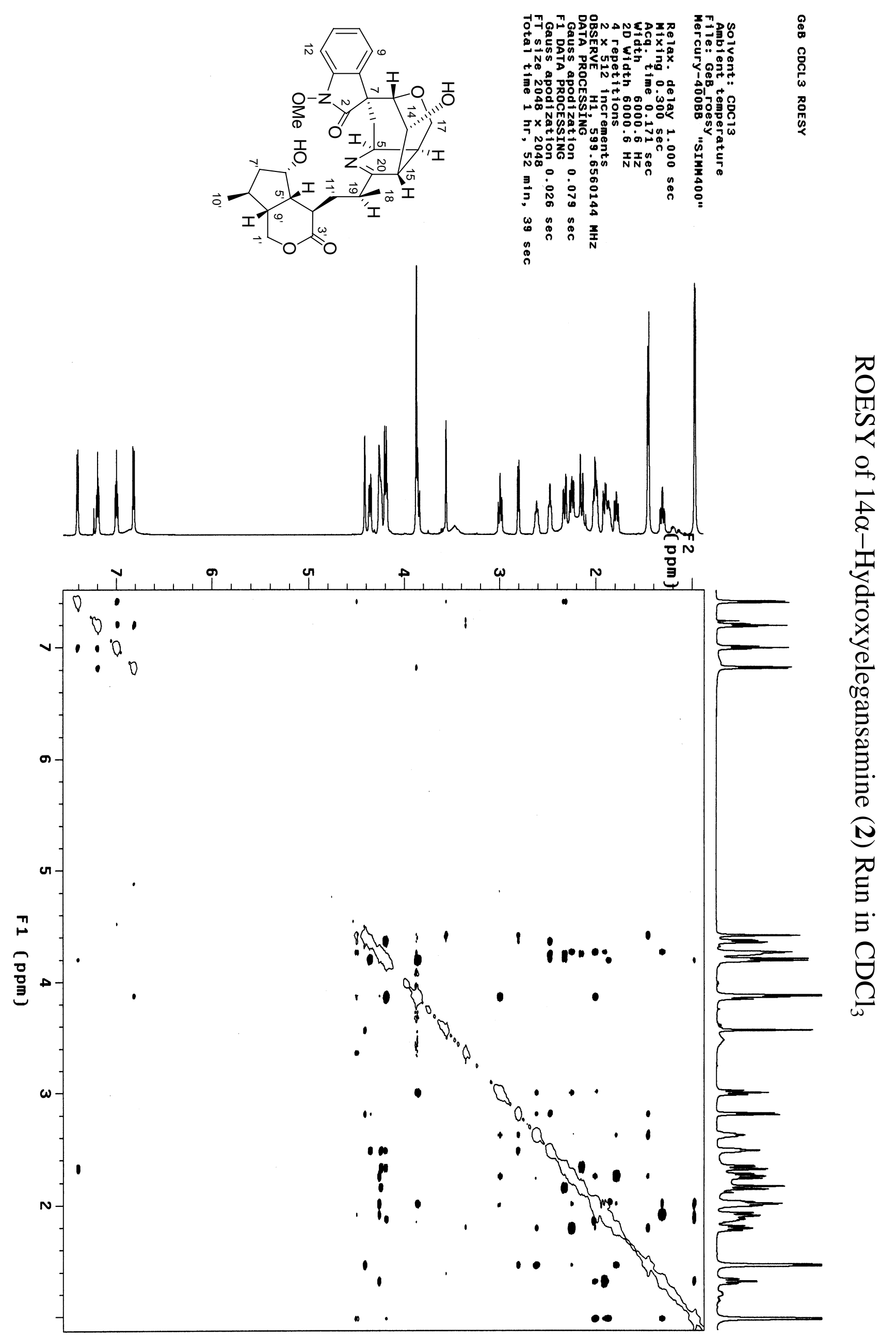




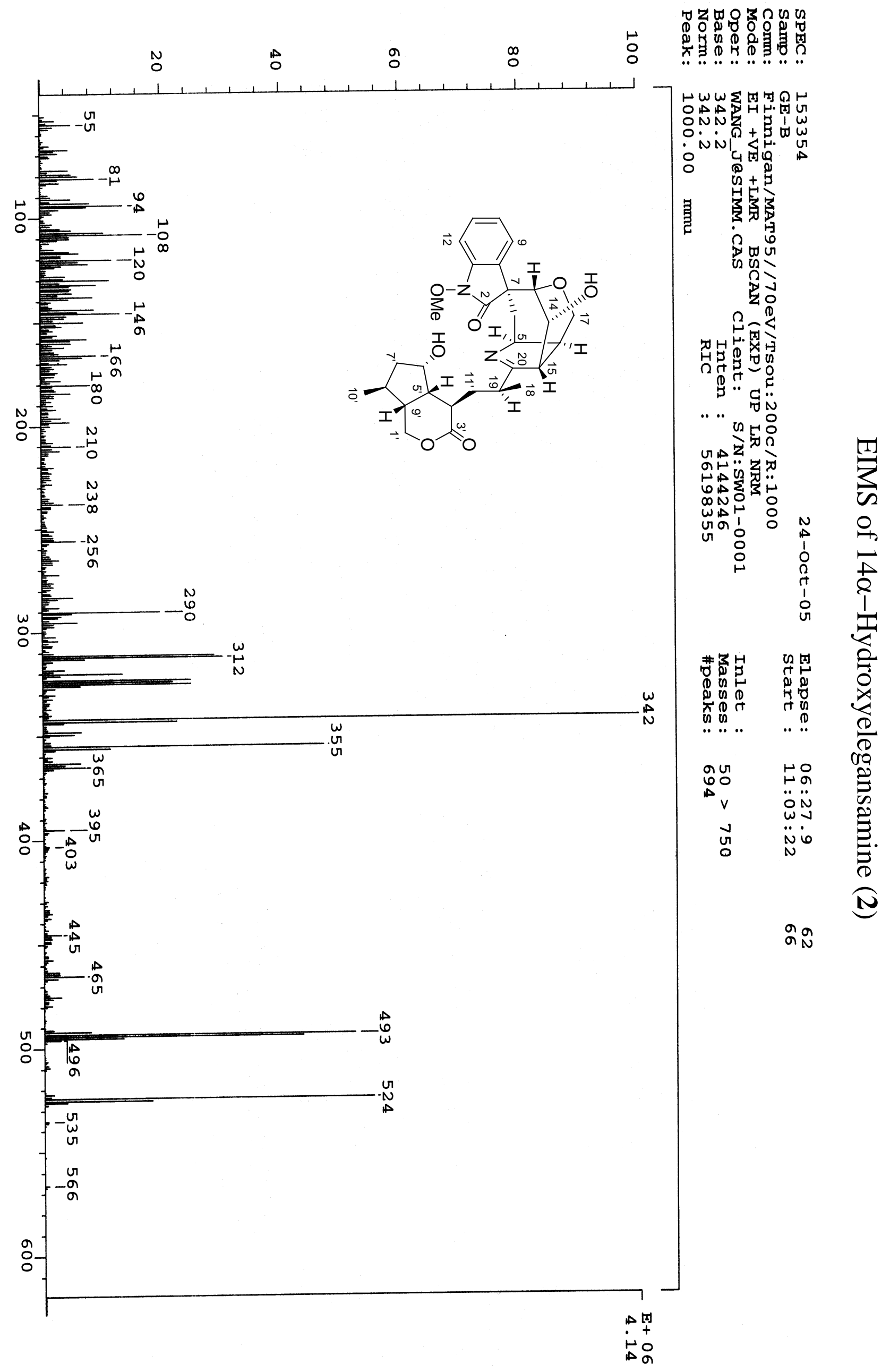




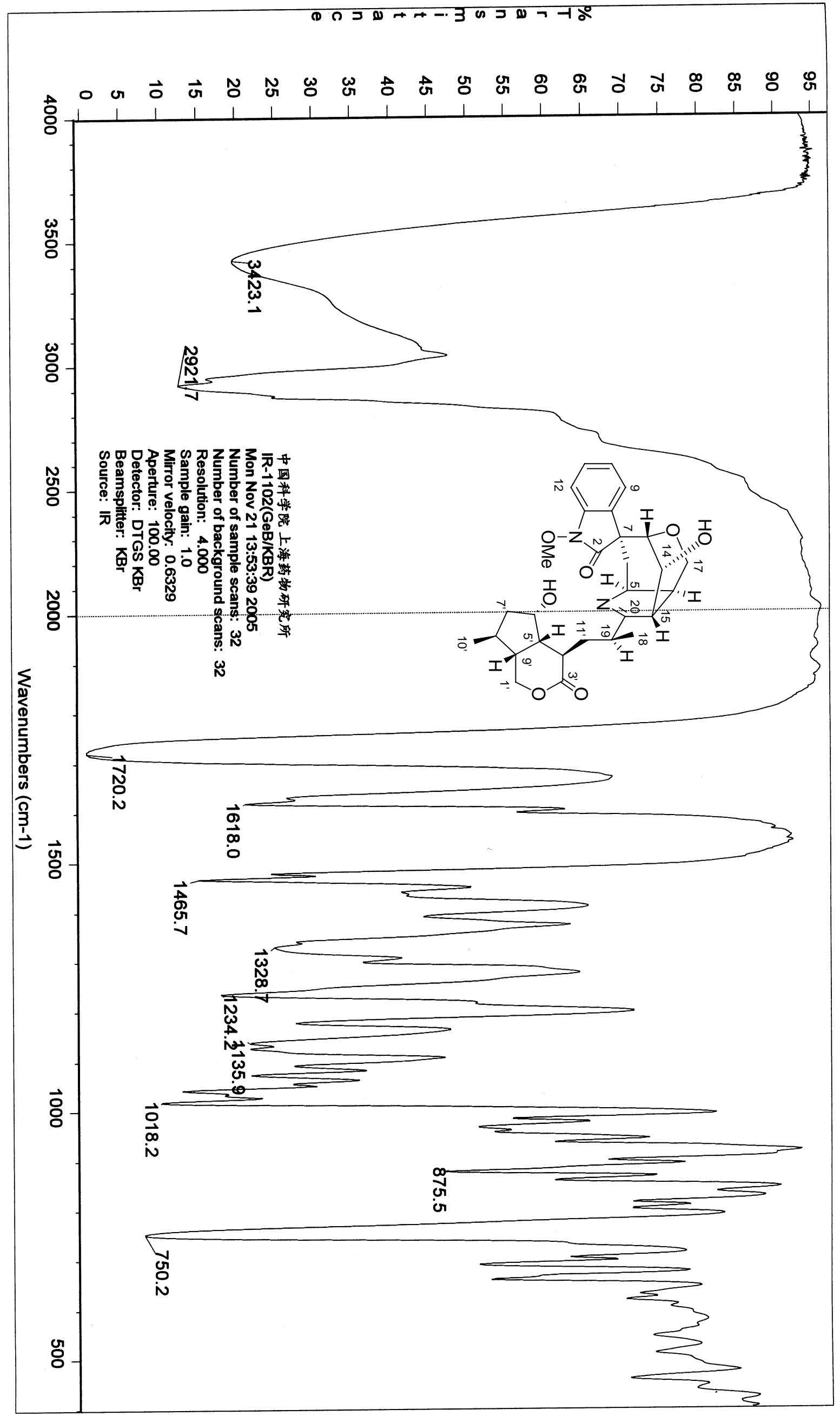

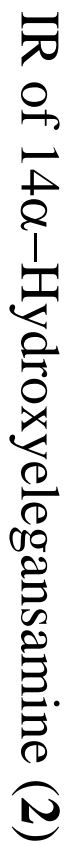



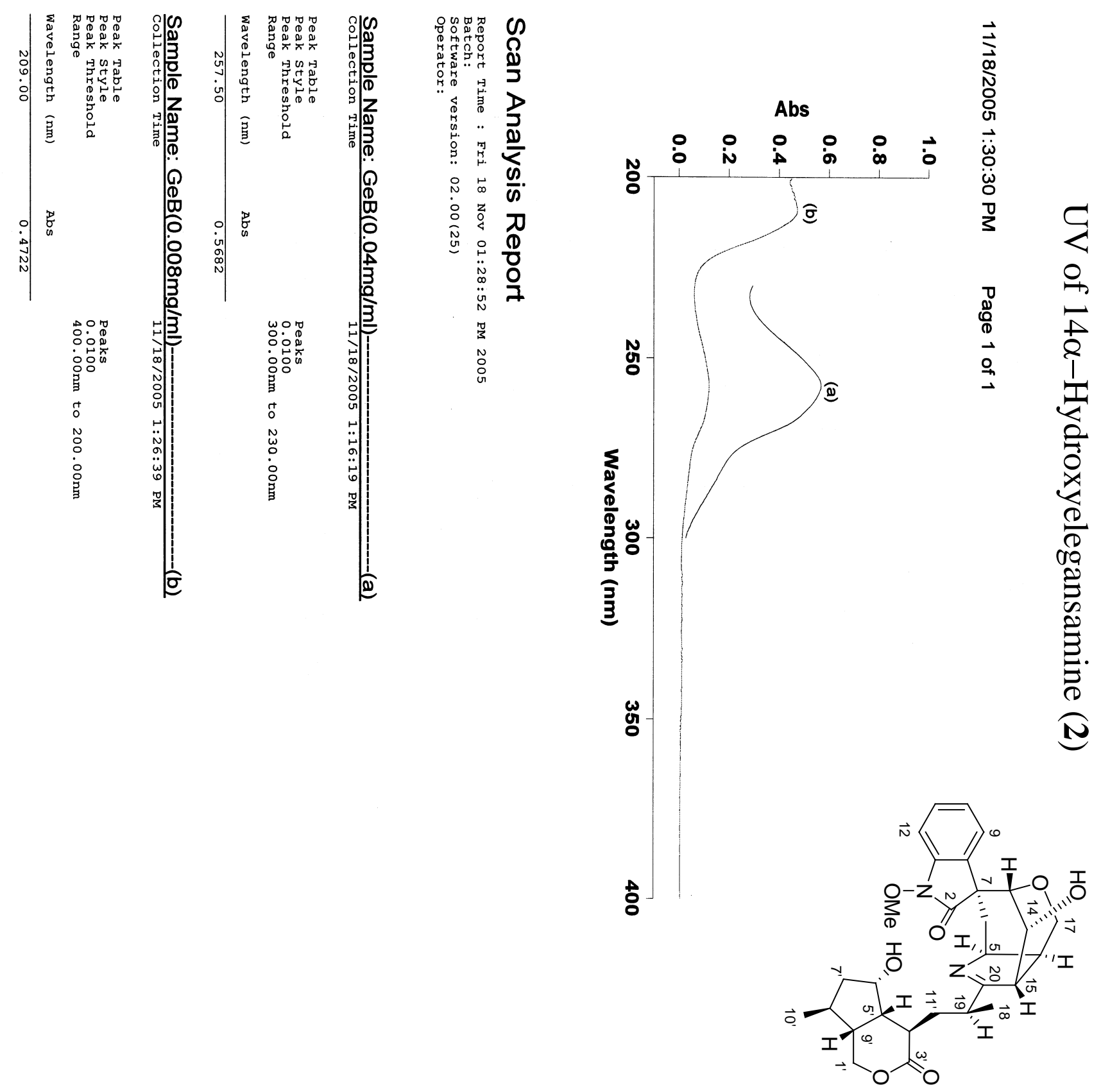

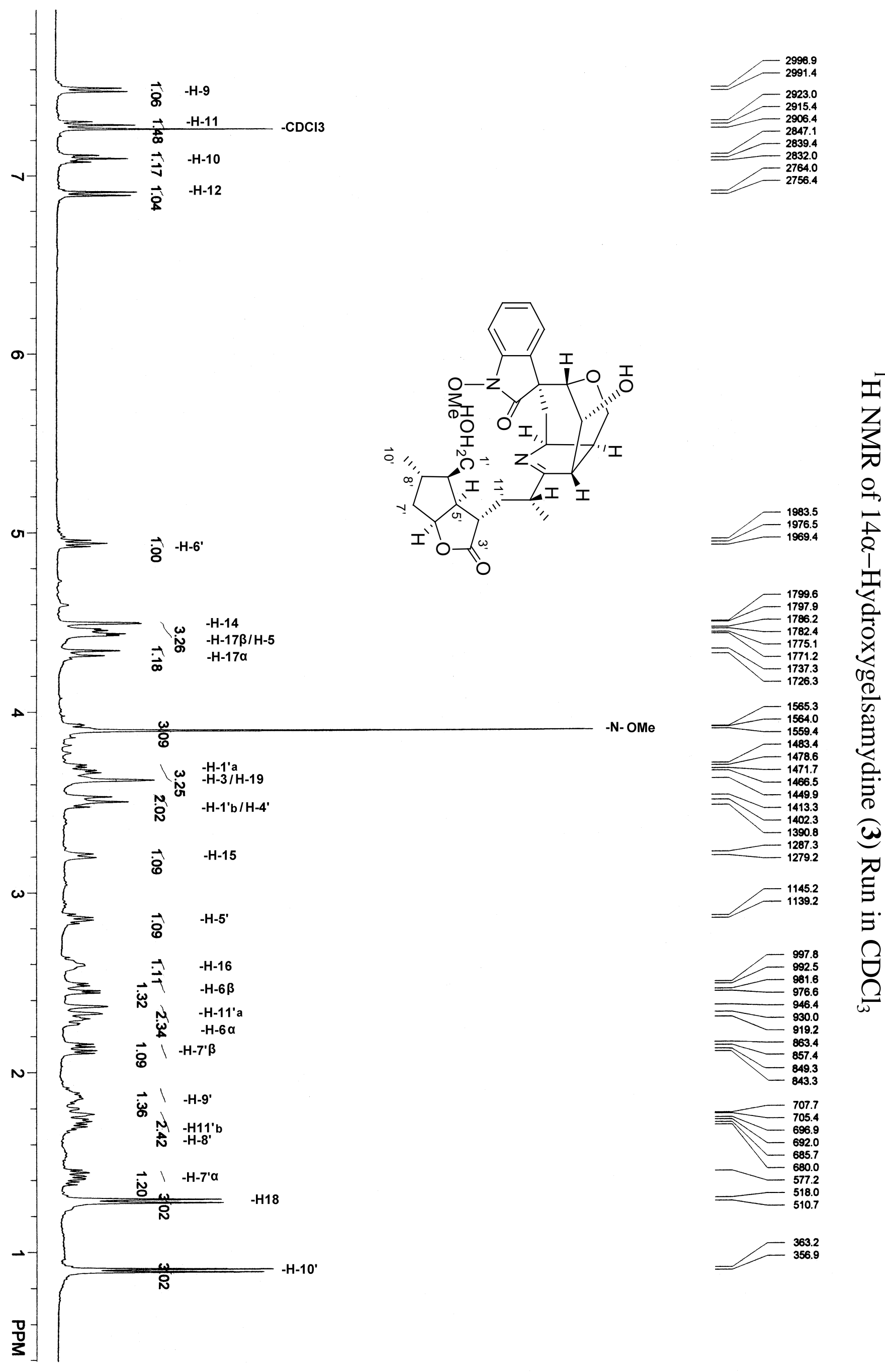


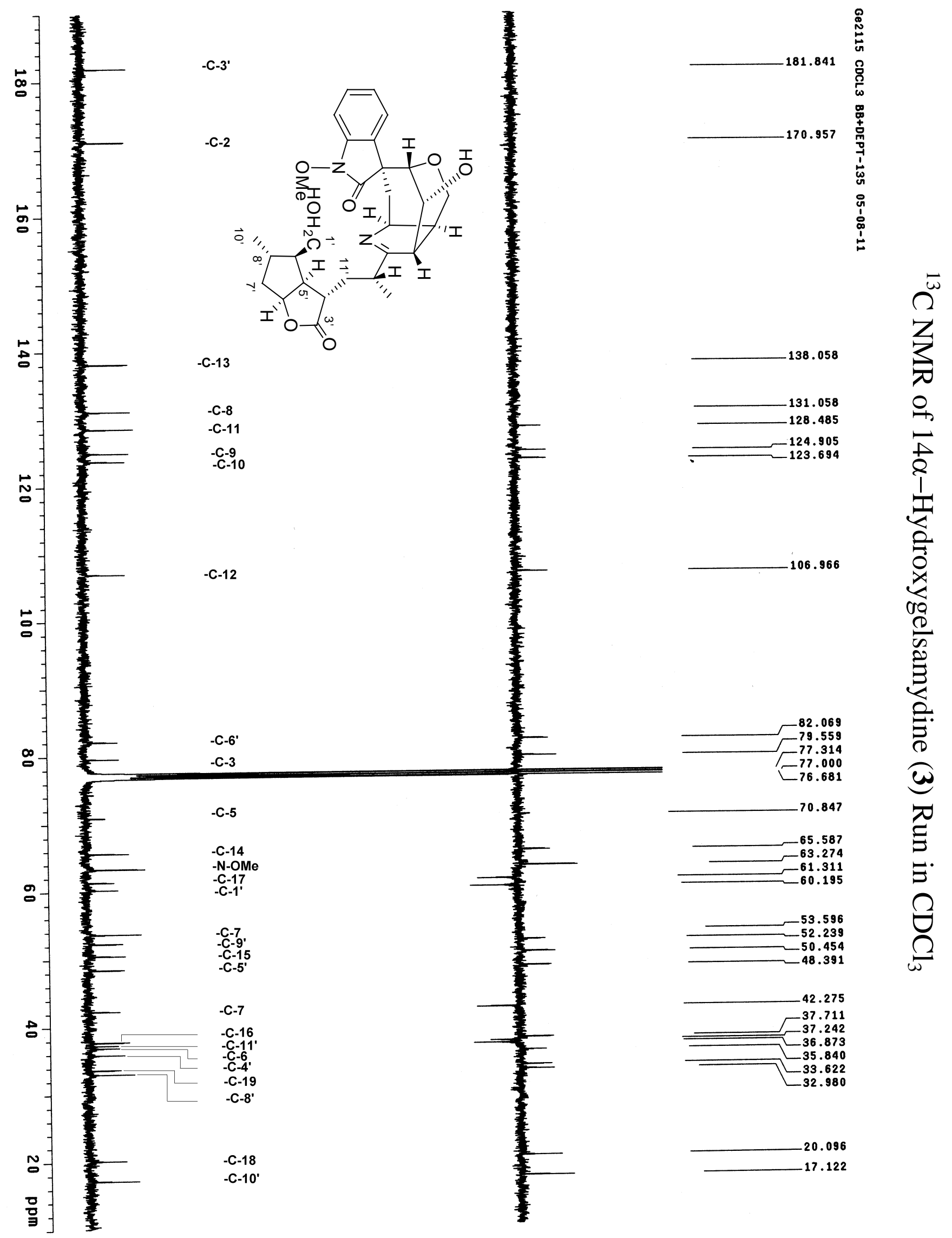



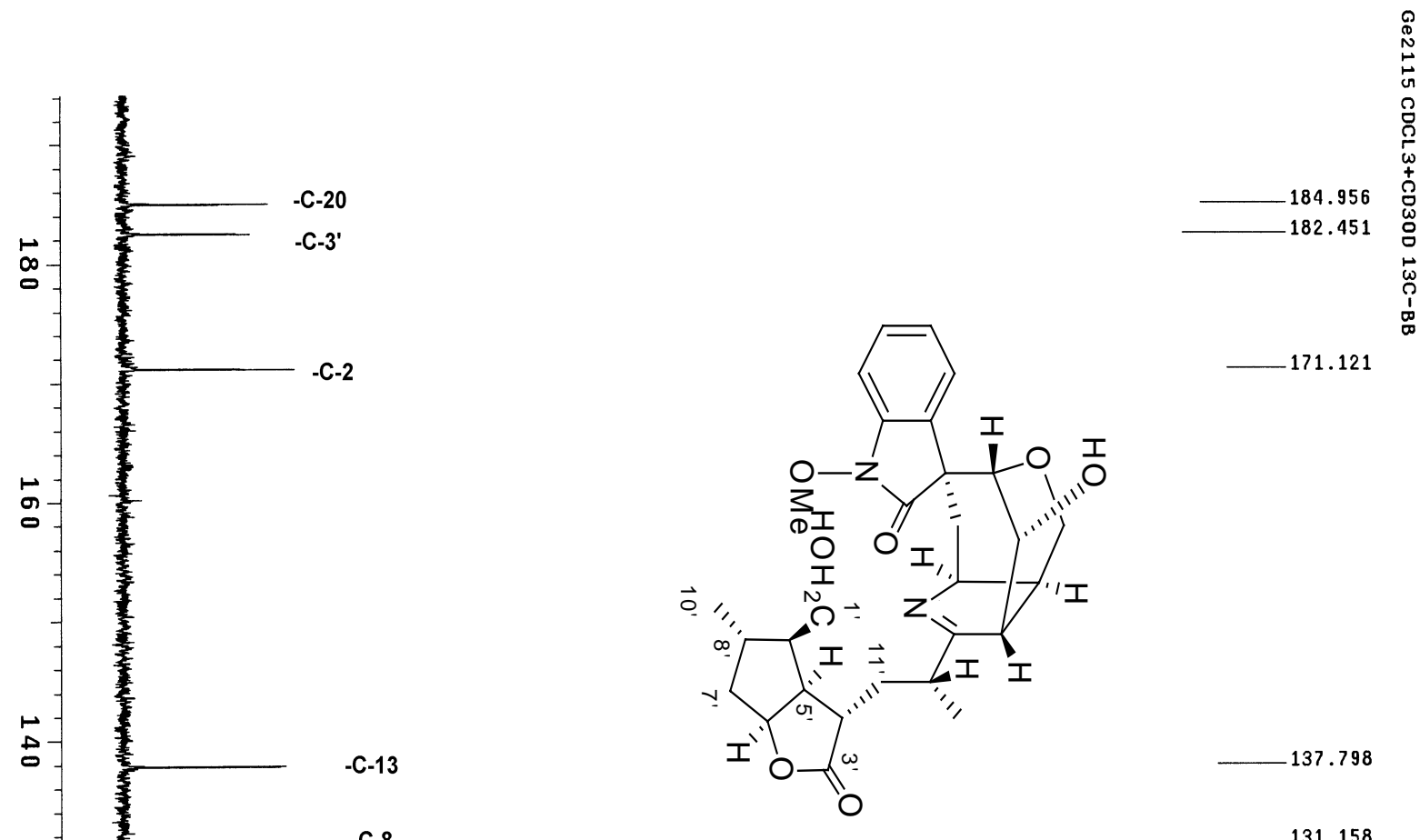

-C-8

$-137.798$

-C-11

131.158

$-128.252$

$-C-C-10$

$-124.919$

믐

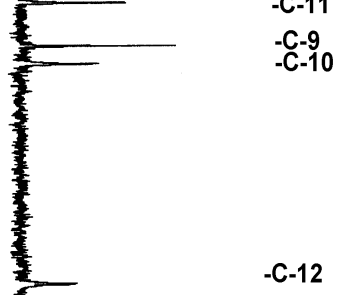

$-106.761$

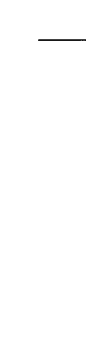

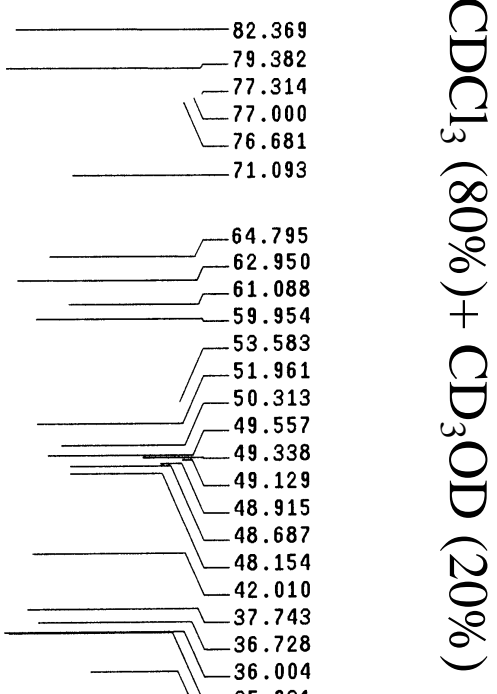

N

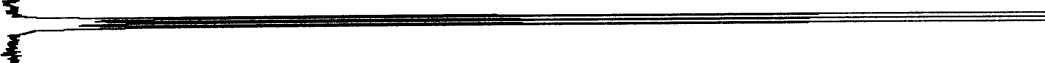

$-C-5$

-C-14

-OMe

a

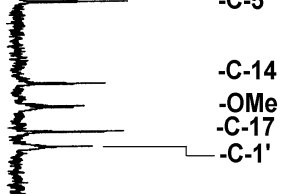

$\therefore$
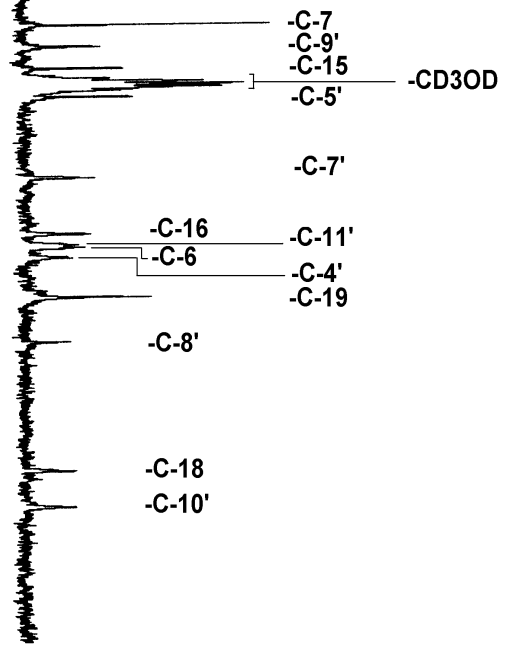

$-C-7^{\prime}$

$-C-11$
$-C-4^{\prime}$
$-C-19$

(1)

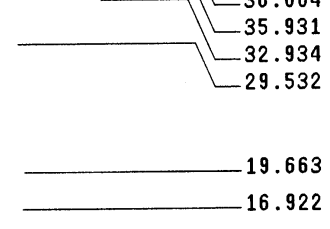




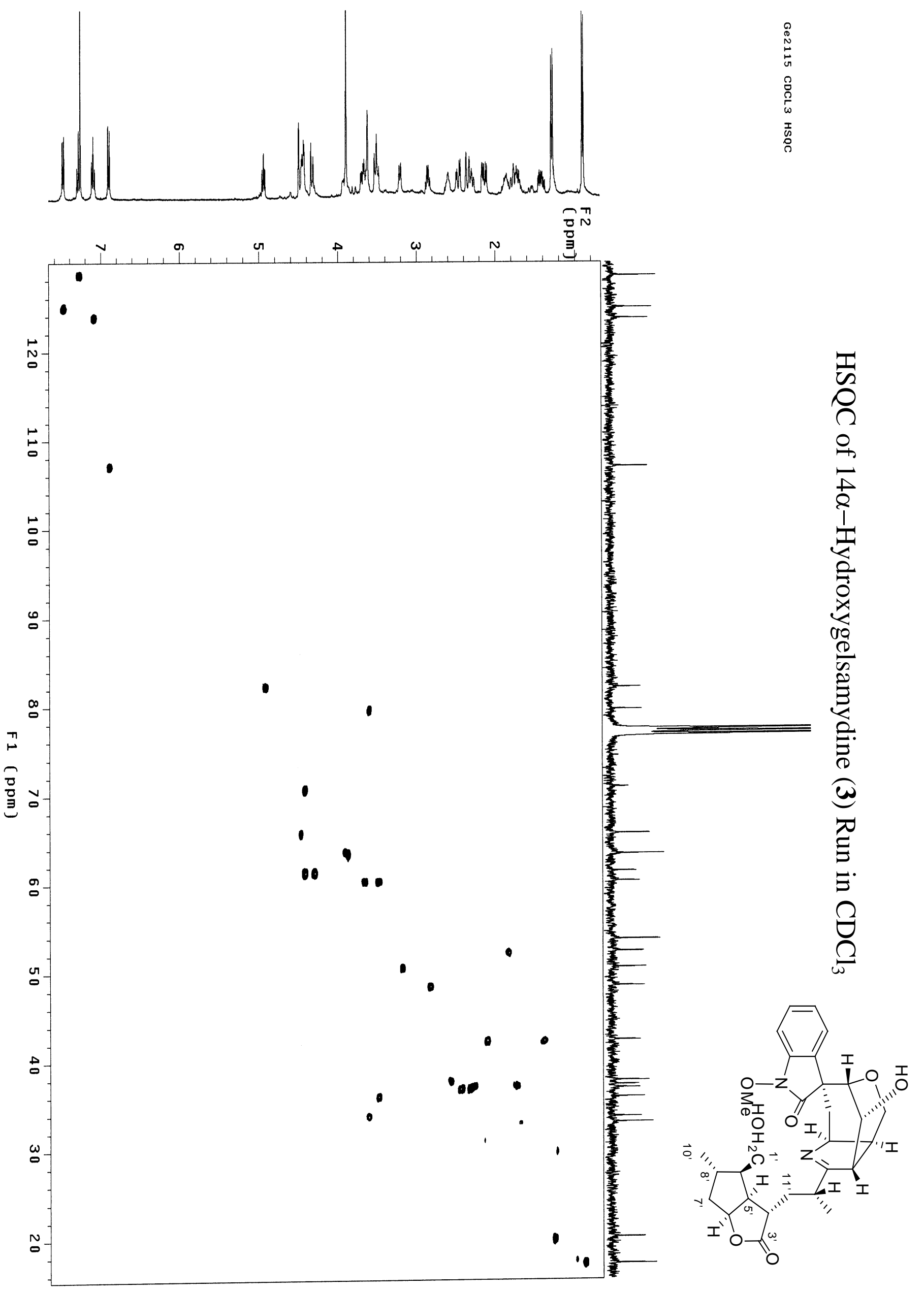




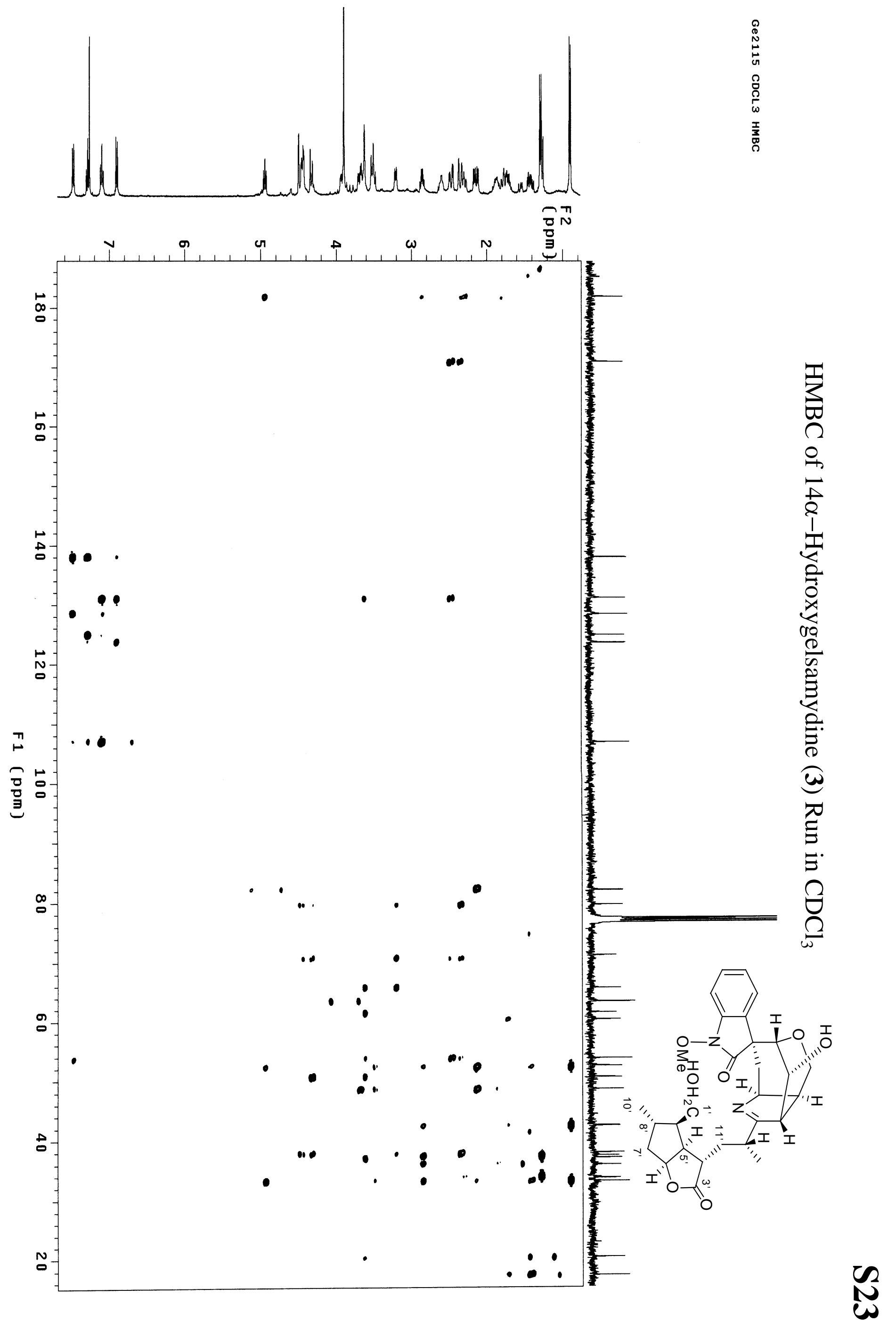




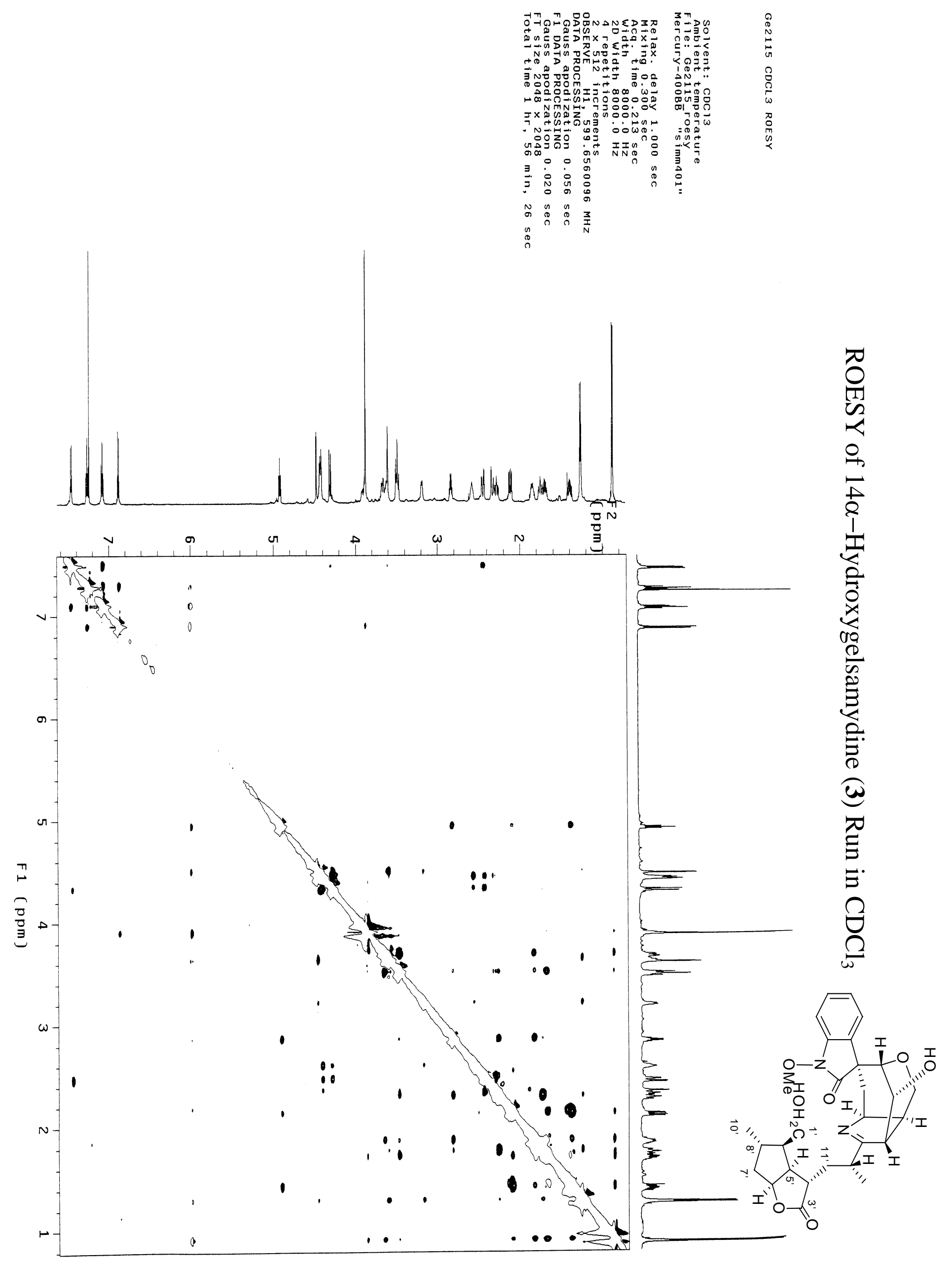




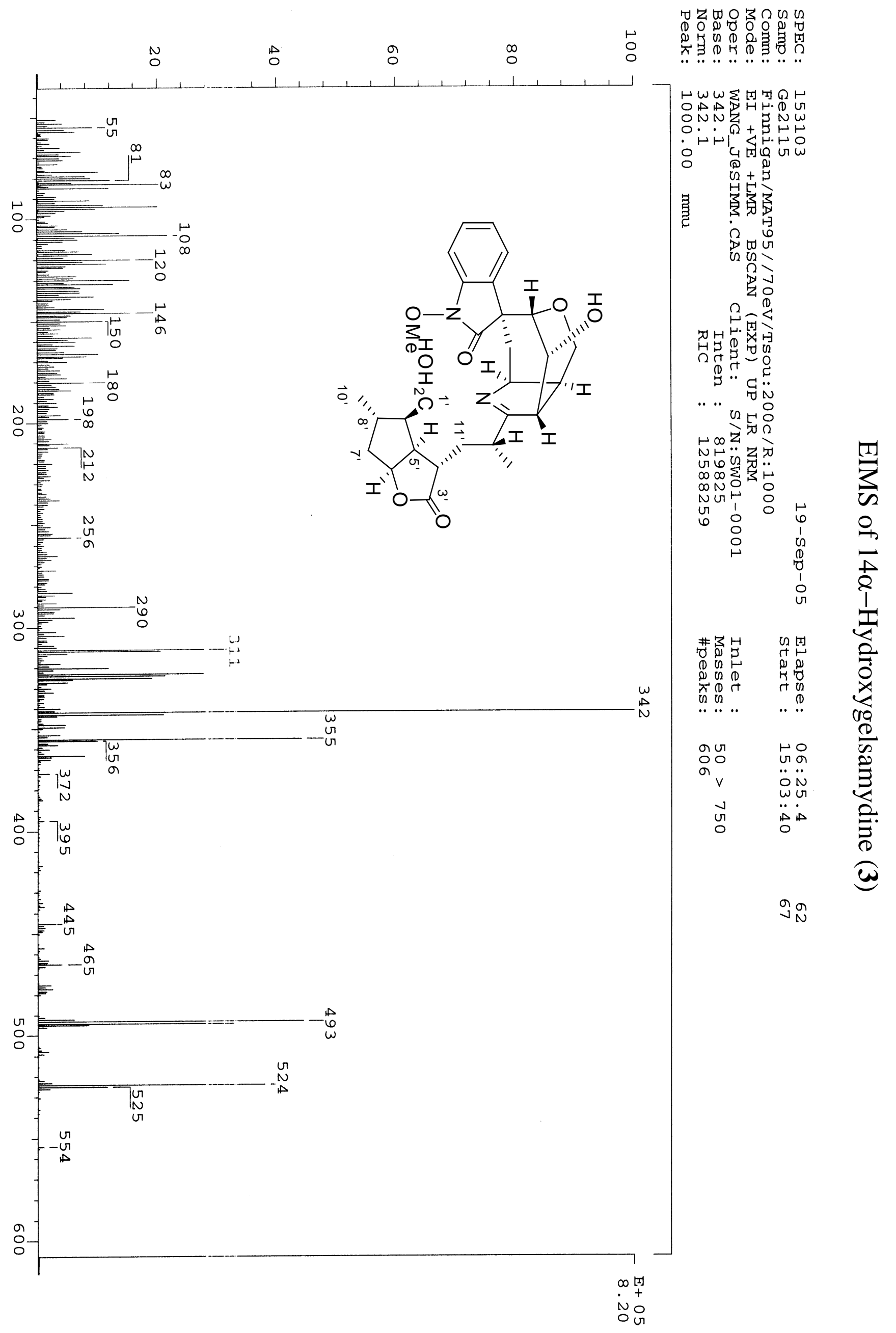




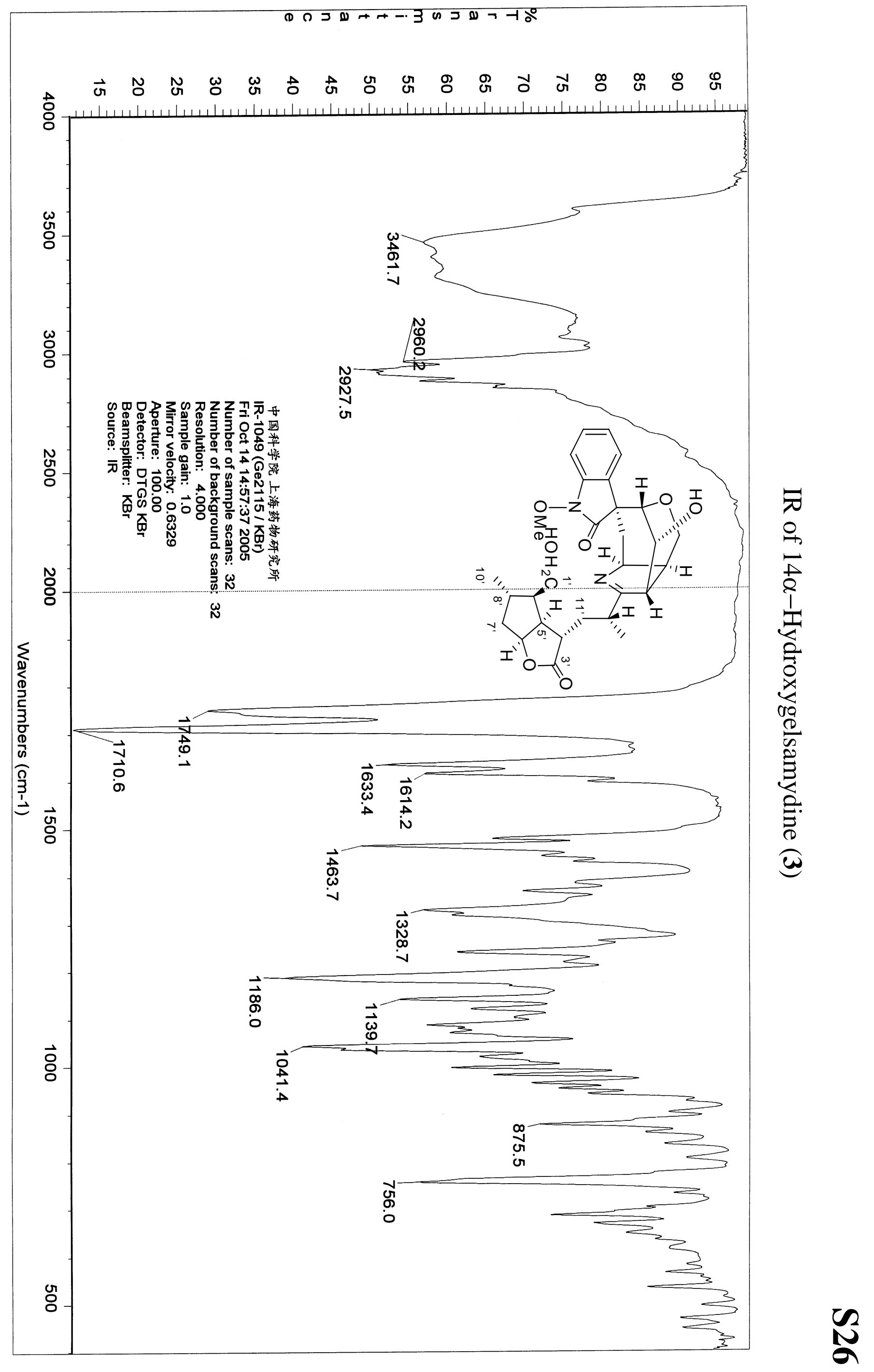



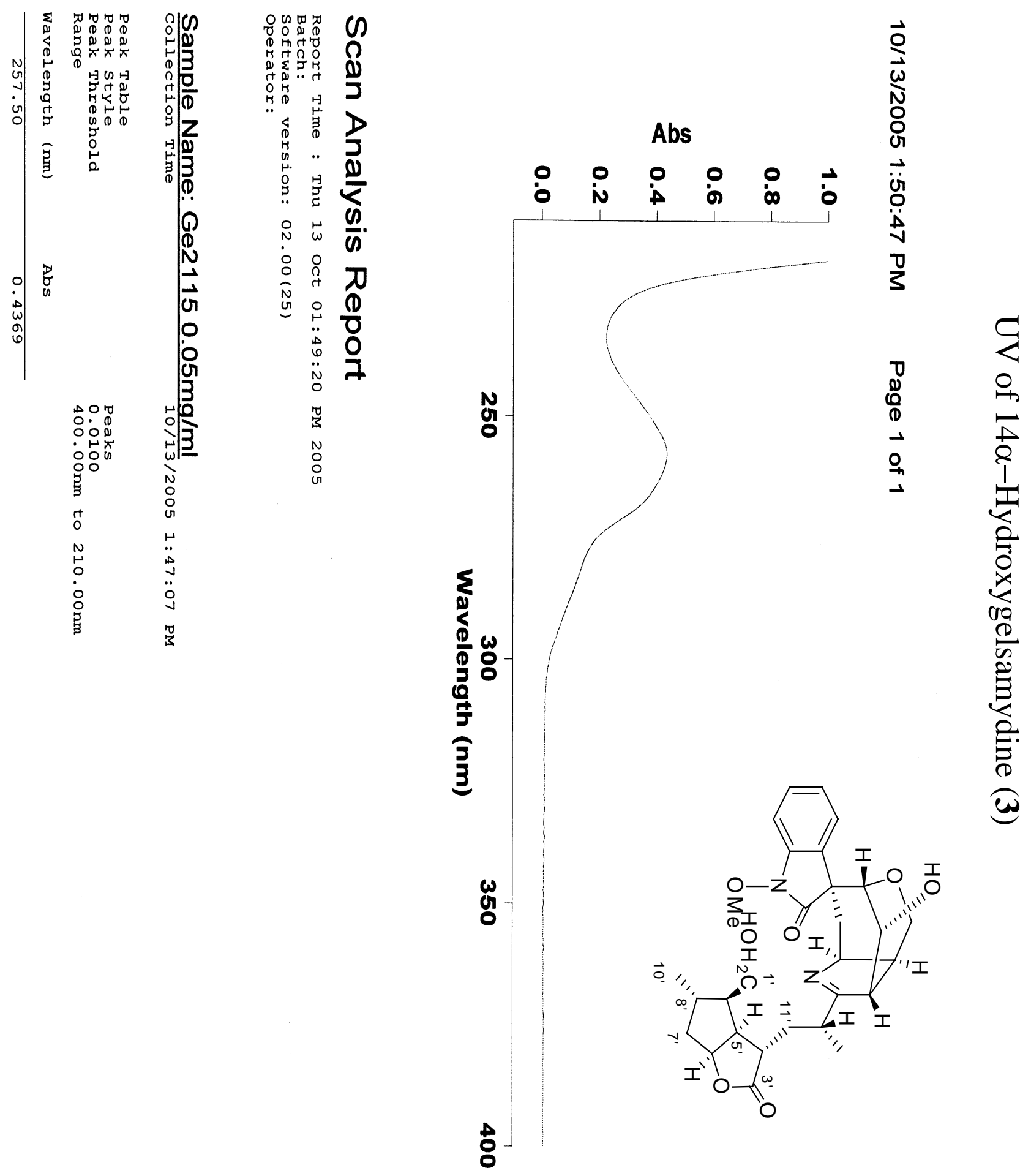


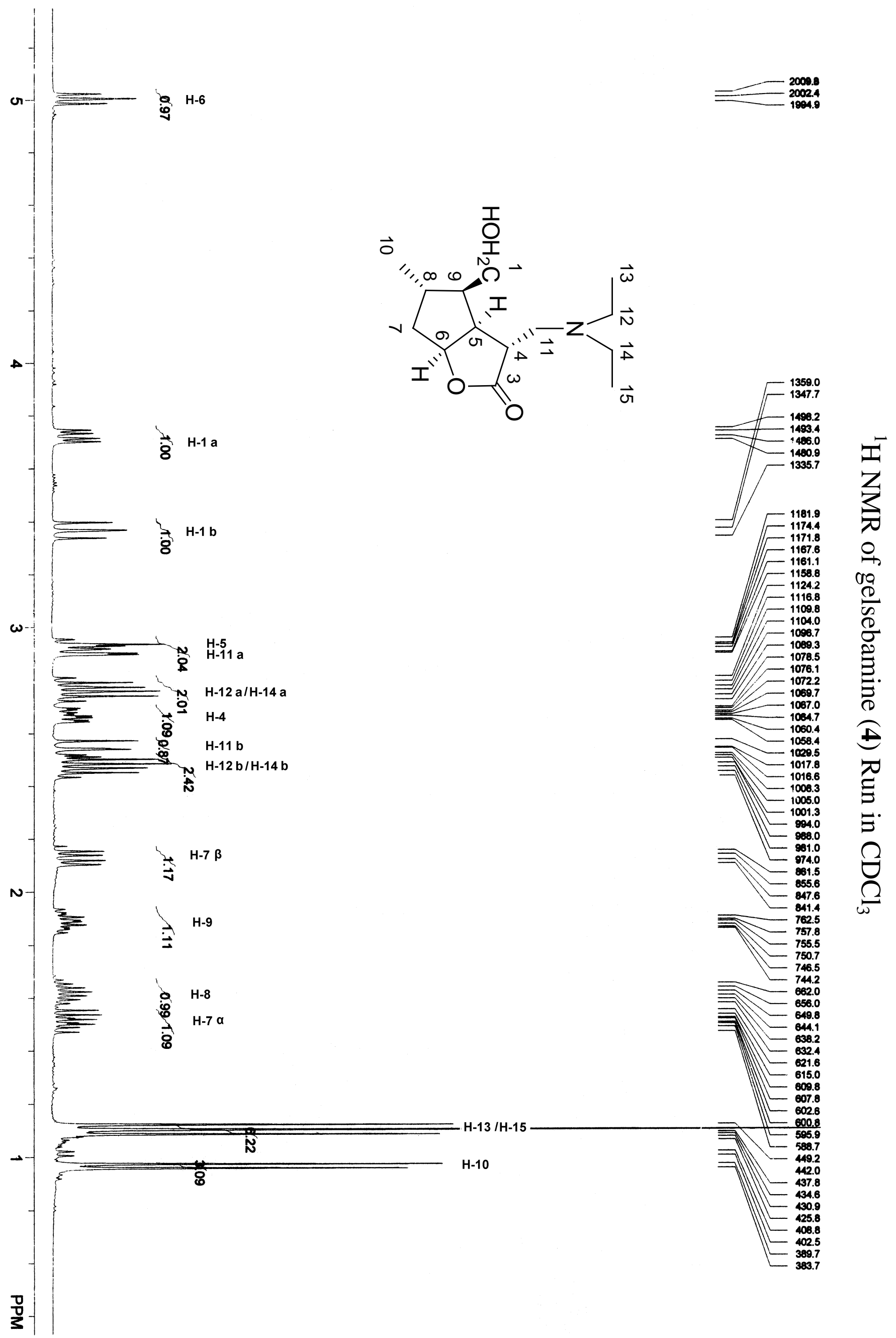



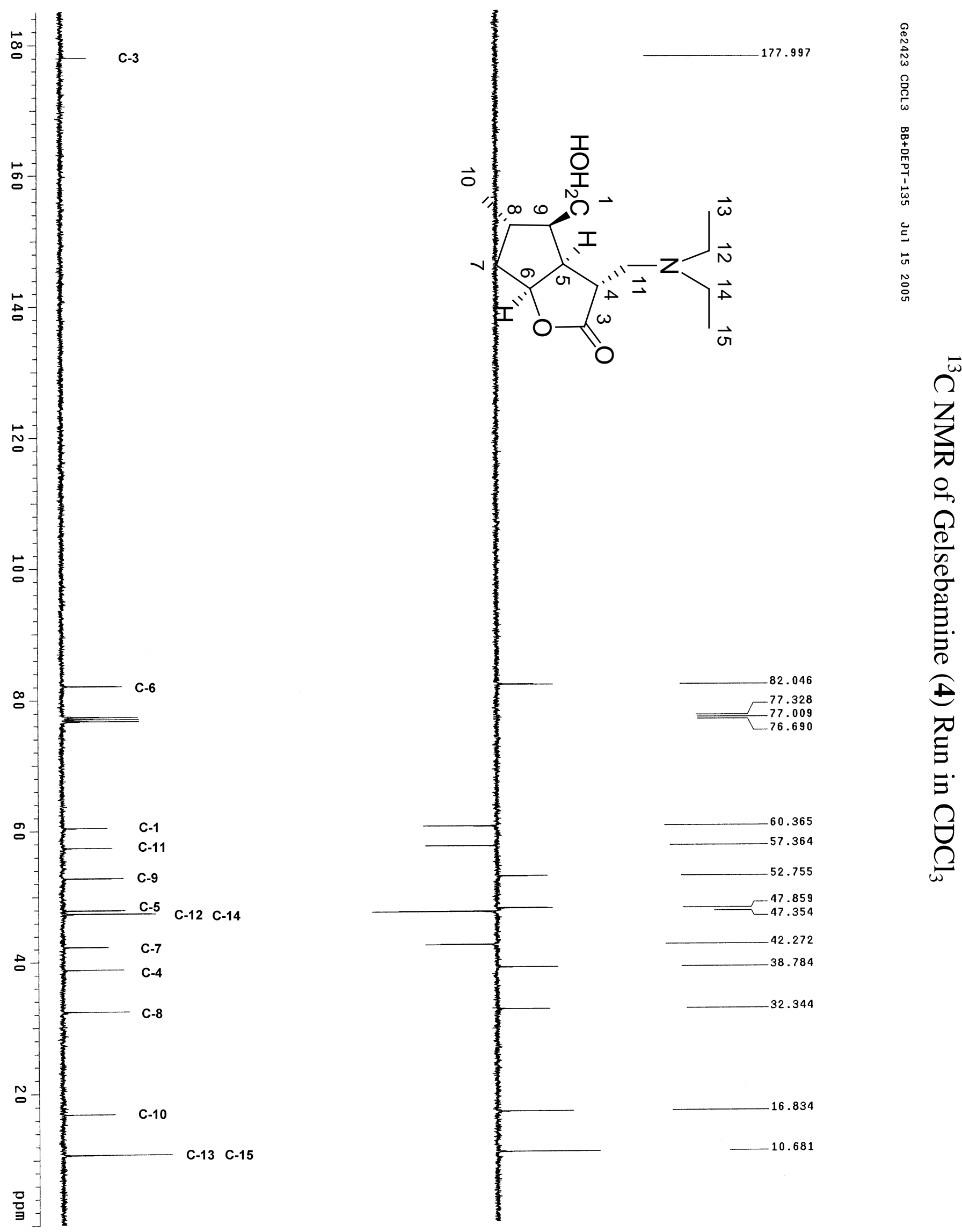


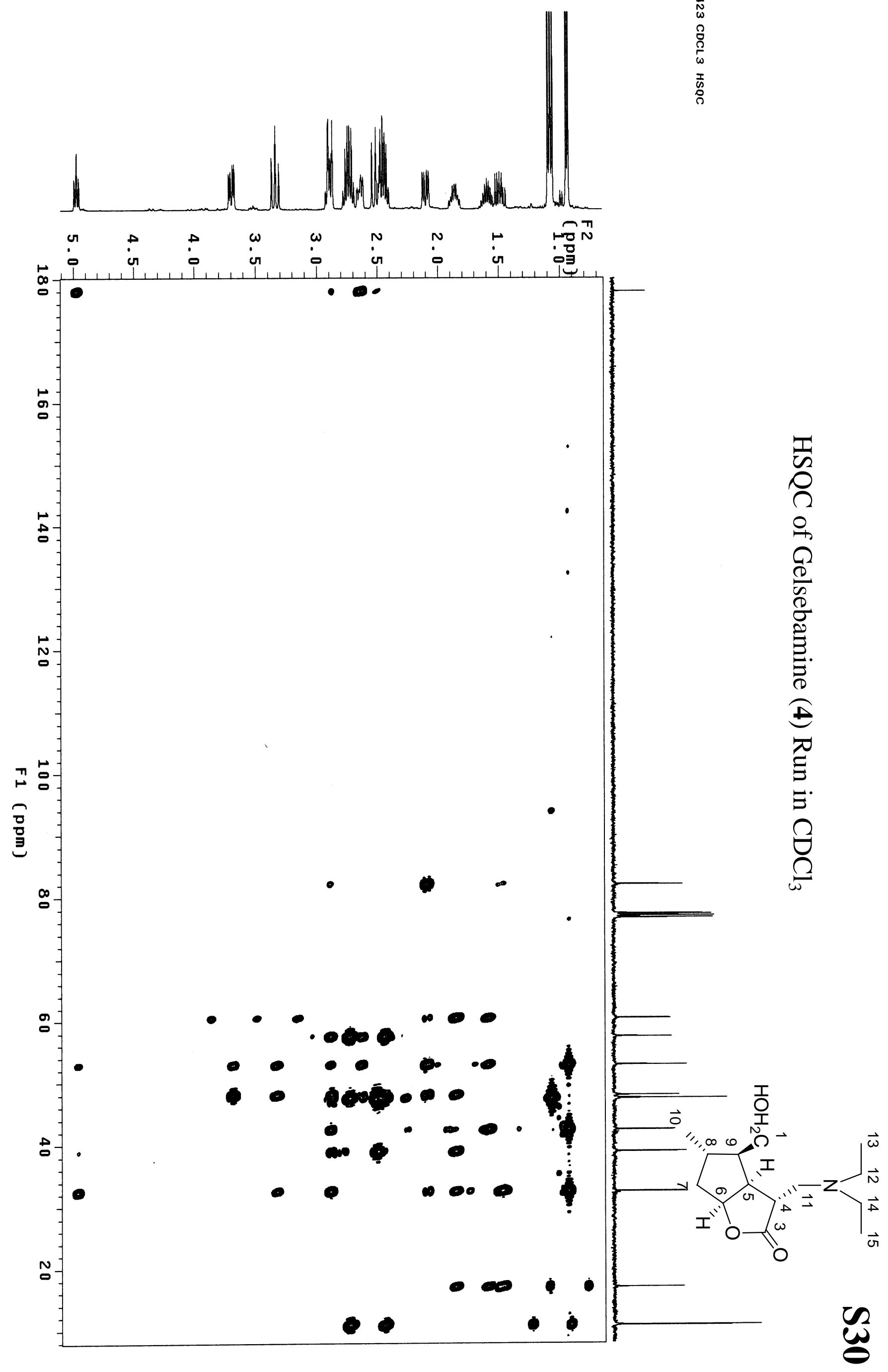




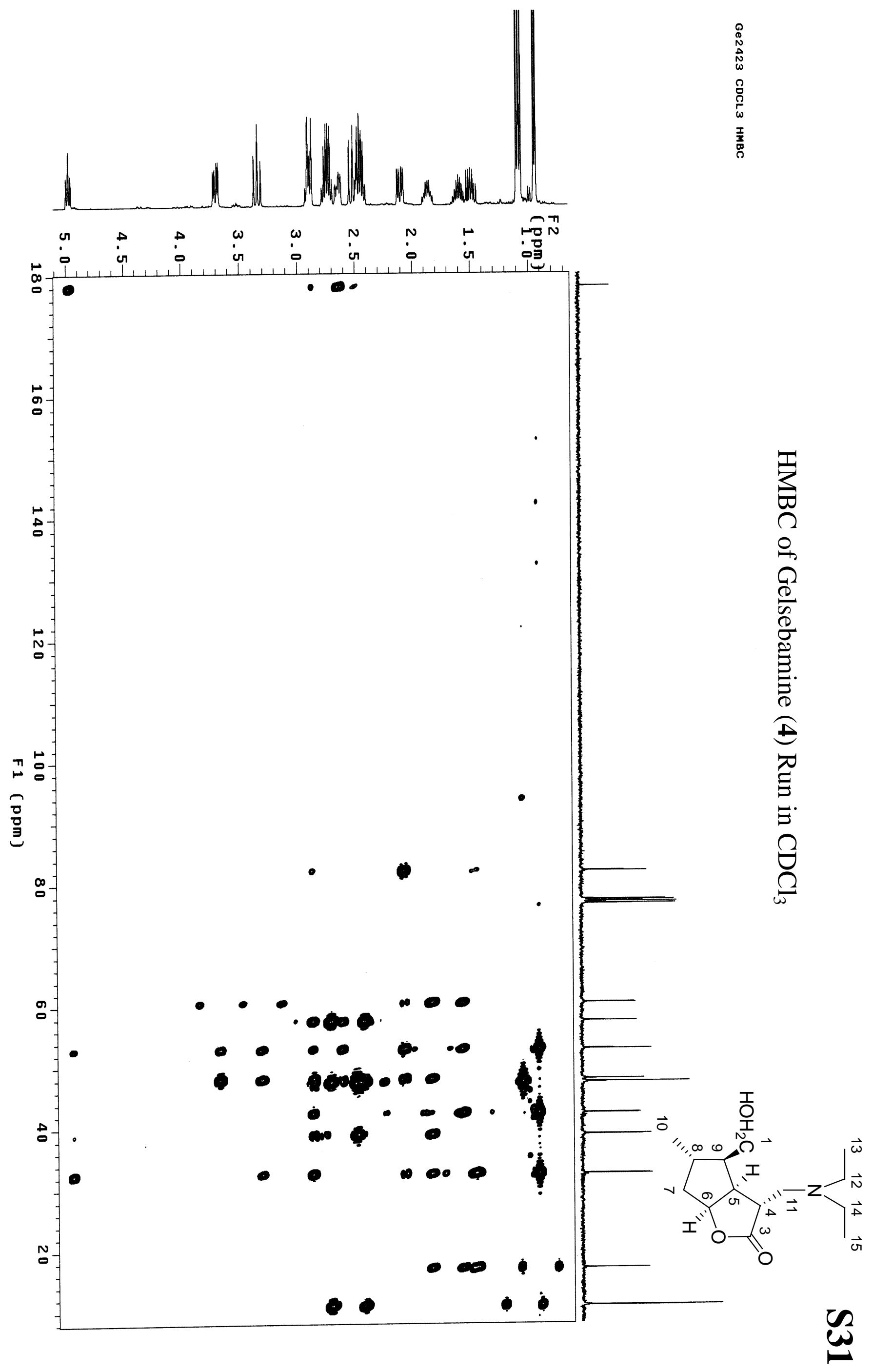




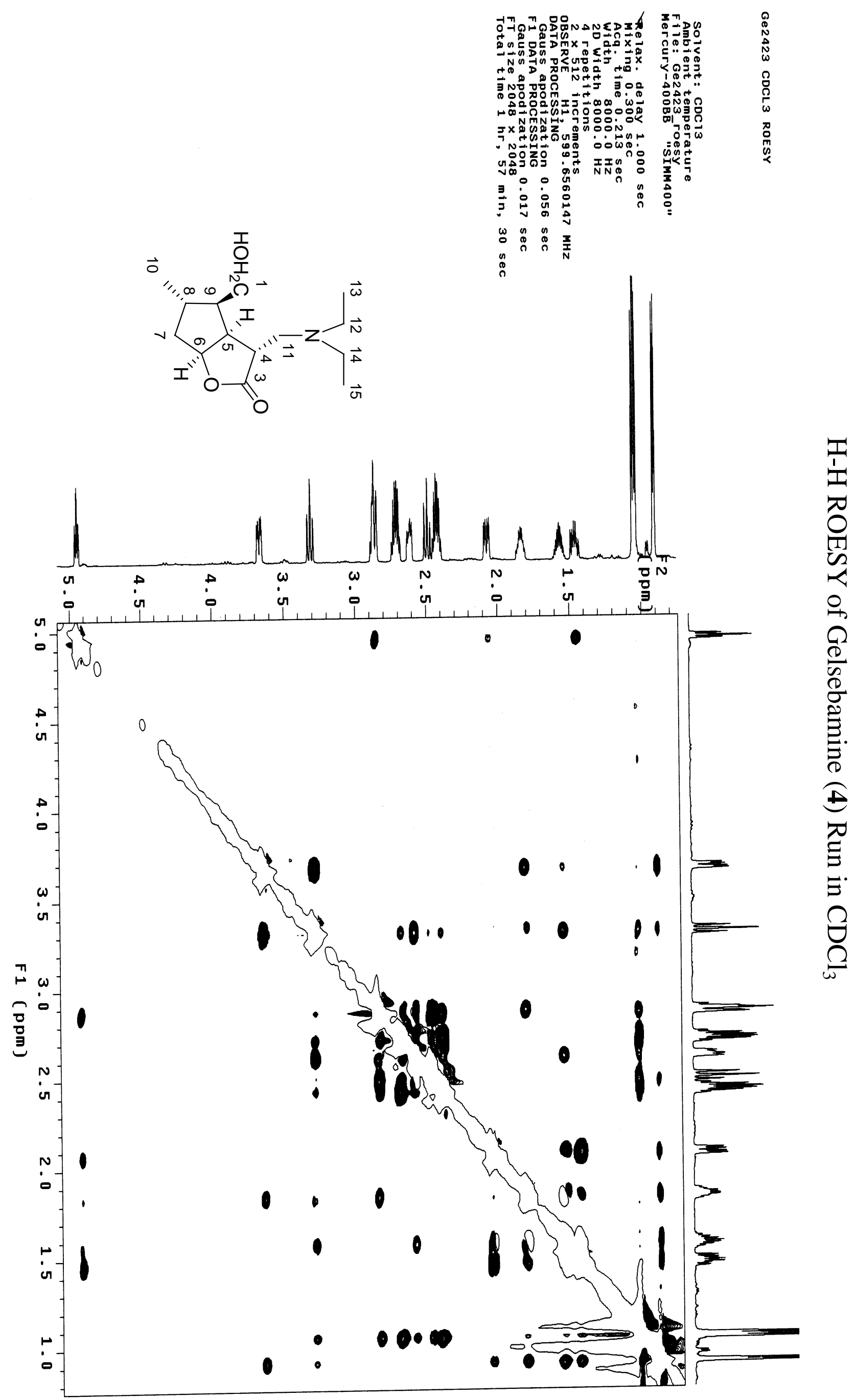




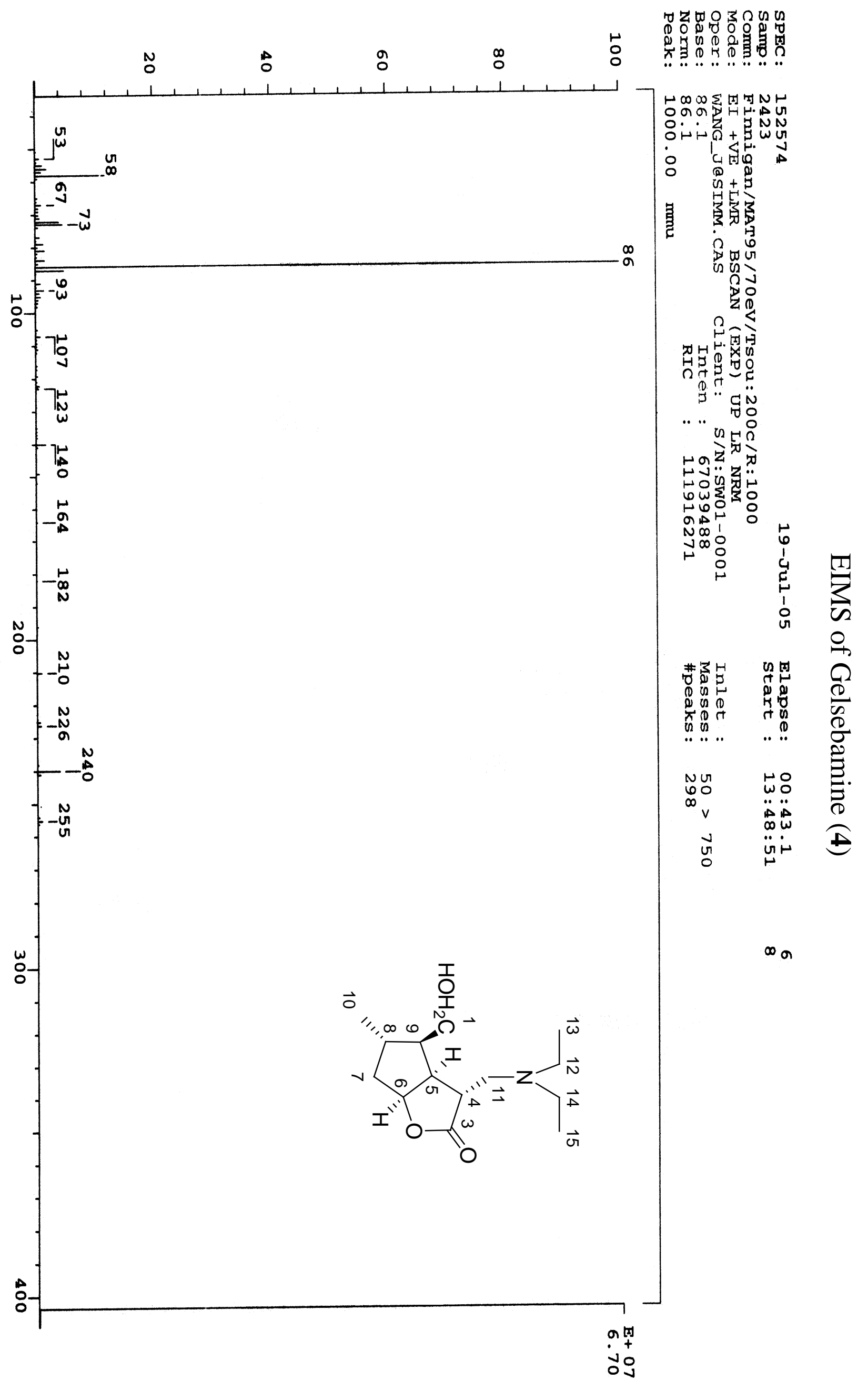




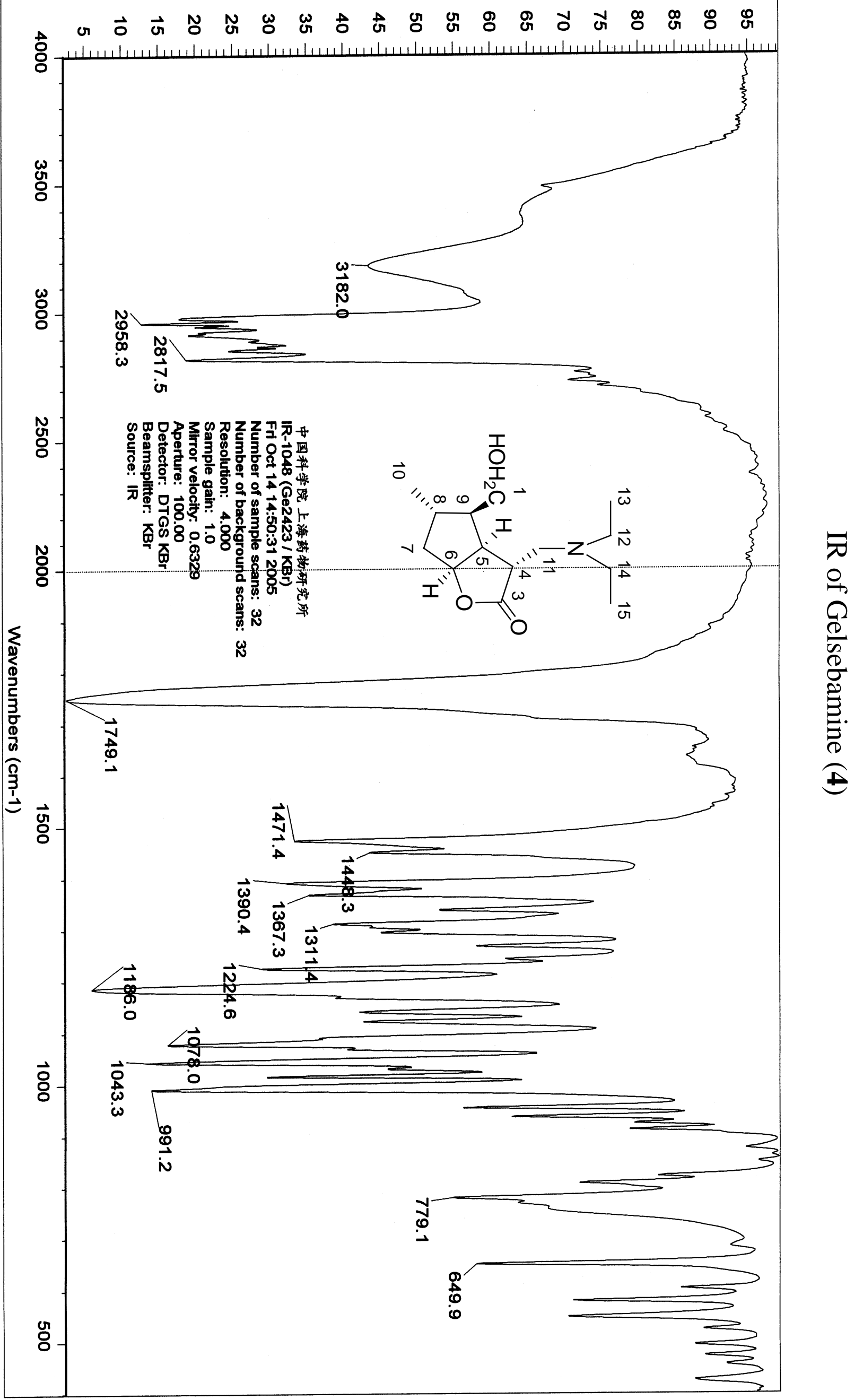


S35

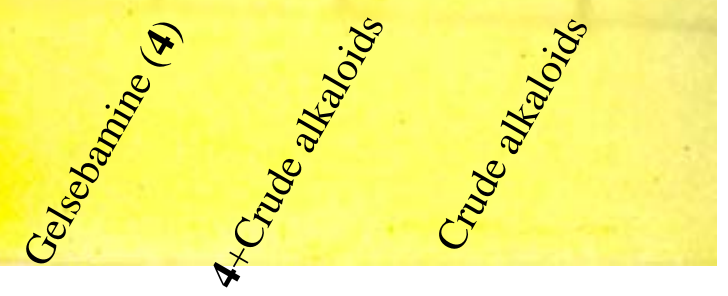

